\title{
Spinal Glutamatergic Neurons Defined by EphA4 Signaling Are Essential Components of Normal Locomotor Circuits
}

\author{
Lotta Borgius, ${ }^{1 \star}$ Hiroshi Nishimaru, ${ }^{2 \star}$ Vanessa Caldeira, ${ }^{1}$ Yuka Kunugise, ${ }^{3}$ Peter Löw, ${ }^{1}$ Ramon Reig, ${ }^{1}$ \\ Shigeyoshi Itohara, ${ }^{4}$ Takuji Iwasato, ${ }^{5}$ and Ole Kiehn ${ }^{1}$ \\ ${ }^{1}$ Department of Neuroscience, Karolinska Institutet, S-17177 Stockholm, Sweden, ${ }^{2}$ Faculty of Medicine and ${ }^{3}$ College of Biological Sciences, University of \\ Tsukuba, 305-8577 Tsukuba, Japan, ${ }^{4}$ RIKEN Brain Science Institute, 351-0198 Wako, Japan, and ${ }^{5}$ National Institute of Genetics, SOKENDAI, 411-8540 \\ Mishima, Japan
}

EphA4 signaling is essential for the spatiotemporal organization of neuronal circuit formation. In mice, deletion of this signaling pathway causes aberrant midline crossing of axons from both brain and spinal neurons and the complete knock-outs (KOs) exhibit a pronounced change in motor behavior, where alternating gaits are replaced by a rabbit-like hopping gait. The neuronal mechanism that is responsible for the gait switch in these KO mice is not known. Here, using intersectional genetics, we demonstrate that a spinal cord-specific deletion of EphA4 signaling is sufficient to generate the overground hopping gait. In contrast, selective deletion of EphA4 signaling in forebrain neurons, including the corticospinal tract neurons, did not result in a change in locomotor pattern. The gait switch was attributed to the loss of EphA4 signaling in excitatory Vglut $2^{+}$neurons, which is accompanied by an increased midline crossing of Vglut $2{ }^{+}$ neurons in the ventral spinal cord. Our findings functionally define spinal EphA4 signaling in excitatory Vglut2 ${ }^{+}$neurons as required for proper organization of the spinal locomotor circuitry, and place these cells as essential components of the mammalian locomotor network.

Key words: $\alpha$-chimaerin; central pattern generator; EphA4; locomotion; spinal cord

\section{Introduction}

The organization of spinal cord networks is orchestrated by a number of molecular determinants that control cell fate as well as axonal guidance and projection. An essential check point for developing axons is their interaction with midline cues that will direct them to either cross the midline and become commissural or stay on the same side and become ipsilaterally projecting neurons. A number of axon guidance molecules participates in control of the midline crossing (Tessier-Lavigne, 2002; Chédotal, 2011) and deletion of these molecules may lead to aberrant axonal wiring and implications for behavior. Prominent examples are deletion of the Robo-Slit (Sabatier et al., 2004) or DCCNetrin-1 signaling pathways (Stein et al., 2001; Fawcett et al., 2007) that lead to a rabbit-like hopping gait (Rabe et al., 2009).

\footnotetext{
Received Nov. 28, 2013; revised Jan. 23, 2014; accepted Jan. 29, 2014

Author contributions: L.B., H.N., and 0.K. designed research; L.B., H.N., V.C., Y.K., P.L., and R.R. performed research; S.I. and T.I. contributed unpublished reagents/analytic tools; L.B., H.N., V.C., Y.K., P.L., and O.K. analyzed data; L.B., H.N., and O.K. wrote the paper.

This work was supported by the Torsten and Ragnar Söderberg Foundations, Swedish Medical Research Council, a DP award from Karolinska Institutet, an advanced ERC grant to 0.K., a KAKENHI (No. 25115702, 23500460) and NIG Cooperative Research Program (2012-A11) to H.N., a FIRST program to S.I., and KAKENHI (No. 22115009) and Uehara Memorial Foundation to T.I. We thank Binhai Zheng for the EphA4 conditional mouse strain, Shota Katori for the ephrinB3 probe, Ann-Charlotte Westerdahl for extensive work on the protocols for genotyping, and Natalie Sleiers and Miyo Kakizaki for animal breeding and participating in the in vivo experiments.

*L.B. and H.N. contributed equally to this work.

The authors declare no competing financial interests.

Correspondence should be addressed to Dr. Ole Kiehn, Mammalian Locomotor Laboratory, Department of Neuroscience, Retzius väg 8, Karolinska Institutet, S-17177 Stockholm, Sweden. E-mail: Ole.Kiehn@ki.se.

DOI:10.1523/JNEUROSCI.4992-13.2014

Copyright $\odot 2014$ the authors $\quad 0270-6474 / 14 / 343841-13 \$ 15.00 / 0$
}

Disruption of the EphA4 signaling pathway, including deletion of the axon guidance receptor EphA4 (Dottori et al., 1998; Kullander et al., 2003), the chemo-repellent EphrinB3 (Yokoyama et al., 2001), or downstream molecules in the EphA4-EphrinB3 signaling pathway such as the $\alpha$-chimaerin $(\alpha$-Chn) or Nck (Egea et al., 2005; Beg et al., 2007; Fawcett et al., 2007; Iwasato et al., 2007; Wegmeyer et al., 2007) also leads to a hopping phenotype. The EphA4 receptor is expressed in the expanded tip of growing axons, and is involved in axonal pathfinding. EphrinB3 is localized in the midline of the spinal cord, and is thought to function as a barrier preventing axons expressing EphA4 from crossing the midline. Both EphA4 and $\alpha$-chimaerin are expressed in a wide variety of neurons throughout the CNS, including the spinal cord. Consequently, it is not known which classes of interneurons are affected in the ephA4 or the $\alpha$-Chn knock-out (KO) mice. Evidence suggests that the hopping phenotype seen in complete EphA4 signaling KOs (ephA4 KOs, EphrinB3 KOs, or $\alpha-C h n$ $\mathrm{KOs}$ ) is due to a reconfiguration of the spinal locomotor network caused by aberrant midline crossing of excitatory neurons that normally stay ipsilateral (Kullander et al., 2003; Butt et al., 2005; Beg et al., 2007; Iwasato et al., 2007; Wegmeyer et al., 2007), although a potential contribution of aberrantly crossing corticospinal tract (CST) neurons has also been suggested (Asante et al., 2010). However, a functional demonstration to evaluate these hypotheses is missing.

In the current study, we have used conditional mouse genetics to functionally show that deletion of EphA4 signaling from CST neurons does not change the locomotor pattern, while deletion of 
EphA4 signaling from intrinsic spinal neurons was sufficient to cause a hopping phenotype resembling that of complete EphA4 signaling KOs. In addition, when EphA4 signaling was deleted selectively from excitatory Vglut $2^{+}$neurons, a similar hopping phenotype was observed and was ascribed to a specific increase in axonal midline crossing of Vglut ${ }^{+}$neurons in the ventral spinal cord.

Our results functionally define spinal EphA4 signaling pathways in excitatory Vglut $2{ }^{+}$neurons as important for appropriate wiring of the locomotor network and show that disrupted pathfinding of this population leads to a pronounced change in the locomotor phenotype, suggesting that these interneurons play a pivotal role in the spinal locomotor network.

\section{Materials and Methods}

Animals. All experiments were approved by the local ethical committees. The following transgenic lines were described previously: Vglut2::Cre (Borgius et al., 2010), Hoxb8::Cre (Witschi et al., 2010), Emx1::Cre (Gorski et al., 2002; Iwasato et al., 2008), $\alpha$-chimaerin $\mathrm{KO}$ (Iwasato et al.,

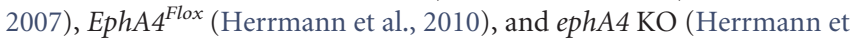
al. 2010). In the conditional $\alpha$-chimaerin ${ }^{\text {Flox }}\left(\alpha-C h n^{\text {Flox }}\right)$, exons 9 and 10 of $\alpha$-chimaerin are flanked by loxP sites and an excision leads to a nonfunctional allele. This mouse will be described later (T. Iwasato and S. Itohara, 2014).

To avoid germ-line recombination we first crossed either Vglut2::Cre; Emx1::Cre or Hoxb8::Cre mice with ephA4 $\mathrm{KO}$ or $\alpha$-Chn $\mathrm{KO}$ mice to obtain the double transgenic lines Emx1::Cre; ephA4 ${ }^{-/+}$, Hoxb8::Cre; ephA4 ${ }^{-1+}$, Vglut2::Cre; ephA $4^{-/+}$, Emx $1:: C r e ; \alpha-C h n^{-/+}$, or Vglut2::Cre; $\alpha-\mathrm{Chn}^{-/+}$. These double transgenic mice were then crossed with the homozygous ephA4 $4^{\text {Flox }}$ or $\alpha$-Chn ${ }^{\text {Flox }}$ mice to obtain the conditional KO mice Emx1::Cre; ephA4 $4^{-/ \text {Flox }}$, Hoxb8::Cre; ephA4 ${ }^{-/ \text {Flox }}$, Vglut2::Cre; ephA4 ${ }^{-/ \text {Flox }}$, Emx 1::Cre; $\alpha-C h n^{-/ \text {Flox }}$, and Vglut2::Cre; $\alpha-C h n^{-/ F l o x}$. All animals were genotyped for the specific Cre allele, the ephA4 ${ }^{\text {Flox }}$, or $\alpha-C h n^{\text {Flox }}$ allele and for the ephA4 or $\alpha$-Chn-null allele. Animals of either sex were used for all experiments.

In vivo locomotor experiments. All in vivo data for the ephA4 transgenic mice were recorded using a TSE MotoRater system that captures the natural movement of animals on a runway (Talpalar et al., 2013). The animals were allowed to walk or run freely in a $120 \times 5 \mathrm{~cm}$ glass-walled corridor and were recorded by a high-speed camera at 100 frames/s. For the different ephA4 transgenic mice, at least five mice at ages from 3 weeks up to 3 months were analyzed for each mouse strain, and for each mouse, runs from at least four independent recordings were analyzed using ClickJoint software (TSE). A run was defined by an instantaneous frequency $>2 \mathrm{~Hz}$ for more than three steps. The first or the last step in a run and episodes with lower frequency than $2 \mathrm{~Hz}$ were not included in the analysis. Phase values were calculated as the time of the swing onset of a limb related to the step cycle length of the reference limb in that particular step cycle. One step cycle corresponds to the time from one swing onset until the next swing onset. Phase values between 0.25 and 0.75 were defined as alternation between limbs, while phase values $<0.25$ and $>0.75$ were defined as synchronous activity between limbs. All the in vivo data for the $\alpha$-Chn transgenic mice were collected by allowing the animals to run freely in a custom-built acrylic-walled corridor $(80 \times 10 \mathrm{~cm})$ and recorded by a video camera at 30 frames/s. All steps were detected using video editing software (CyberLink PowerDirector) and analyzed in a similar way as ephA4 transgenic mice. Fisher's exact test of independence was used to test if the number of alternating and synchronous steps was significantly $(p<0.05)$ different at low $(\leq 4 \mathrm{~Hz})$ and high $(>4 \mathrm{~Hz})$ locomotor frequencies. For this analysis, all phase values were divided into low or high frequency, and within these two groups, all phase values were categorized as alternating $(0.25-0.75)$ or synchronous $(\leq 0.25$ and $\geq 0.75)$ activity. The total numbers of phase values within each of these four groups were then placed in $2 \times 2$ " $R \times$ $C$ table" for Fisher's test.

In vitro electrophysiological experiments. Spinal cords from animals aged postnatal day $0-2(\mathrm{P} 0-\mathrm{P} 2)$ were used for in vitro experiments. Animals were anesthetized with isoflurane before decapitation. Thereafter the spinal cord was exposed in cold $\left(4^{\circ} \mathrm{C}\right)$, low $\mathrm{Ca}^{2+}$ Ringer's solution containing the following (in $\mathrm{mm}$ ): $111 \mathrm{NaCl}, 3 \mathrm{KCl}, 11$ glucose, 25 $\mathrm{NaHCO}_{3}, 3.7 \mathrm{MgSO}_{4}, 1.1 \mathrm{KH}_{2} \mathrm{PO}_{4}$, and $0.25 \mathrm{CaCl}_{2}$, gassed with $95 \%$ $\mathrm{O}_{2} / 5 \% \mathrm{CO}_{2}$, $\mathrm{pH}$ 7.4. The isolated spinal cord was placed in a recording chamber (at room temperature) and continuously superfused with normal Ringer's solution of the following composition (in $\mathrm{mm}$ ): $111 \mathrm{NaCl}, 3$ $\mathrm{KCl}, 11$ glucose, $25 \mathrm{NaHCO}_{3}, 1.25 \mathrm{MgSO}_{4}, 1.1 \mathrm{KH}_{2} \mathrm{PO}_{4}$, and $2.5 \mathrm{CaCl}_{2}$, gassed with $95 \% \mathrm{O}_{2} / 5 \% \mathrm{CO}_{2}, \mathrm{pH}$ 7.4. Ventral root (VR) activity was recorded from lumbar (L) VRs using glass suction electrodes filled with Ringer's solution. Signals were amplified, bandpass filtered $(100-1 \mathrm{kHz})$, sampled at $1 \mathrm{kHz}$, digitized, and acquired with pCLAMP software (version 10; Molecular Devices) or Spike2 software (CED) for subsequent off-line analysis.

Chemically evoked locomotor-like activity was induced by continuous bath application of NMDA (5-30 $\mu$; Sigma) plus 5-hydroxytryptamine (5-HT; 5-15 $\mu \mathrm{M}$; Sigma). Descending fiber-evoked locomotor-like activity was induced by placing a large suction electrode over the upper cervical spinal cord and delivering trains ( $60 \mathrm{~s}$ long) of low-frequency $(0.5-2$ $\mathrm{Hz}$, stimulus duration $0.2-1 \mathrm{~ms}$ ) stimuli with strength of $0.1-1 \mathrm{~mA}$ (Talpalar and Kiehn, 2010).

For the midsagittal lesions of the dorsal commissure, preparations (T5-L4) were placed dorsal side up and the midline was cut from the surface to the central canal (CC) with a fine surgical scissor or a razor blade. After electrophysiological recordings, the preparations were fixed in $4 \%$ paraformaldehyde (PFA) in PBS (12-24 h), cryoprotected in 20\% sucrose for $24 \mathrm{~h}$, and sectioned on a vibratome ( $250 \mu \mathrm{m}$ sections, VT1000S; Leica Biosystems) for further anatomical assessment of the lesion.

Analysis of in vitro electrophysiological data. For the analysis of leftright and flexor and extensor coordination 30 locomotor cycles were selected. Circular statistics were used to determine the phase relationship between left (1) and right (r) L2 or L5 VRs and between L2 and L5 VRs on the same side during the locomotor-like rhythmic activity as previously described (Kjaerulff and Kiehn, 1996; Iwasato et al., 2007; Talpalar et al., 2013). The phase values $(\Phi)$ of the left VR burst onsets were calculated with regard to right VR onsets (left-right coordination) and those of the extensor (L5) VR were calculated with regard to flexor (L2) VR onsets (flexor-extensor coordination). The mean phase $(\Phi)$, expressed in angular degrees, refers to the interval between the onset of a cycle in one root and the onset of the corresponding cycle on the other root, divided by the period (Kjaerulff and Kiehn, 1996). The mean phase is indicated by the direction of the vector originating from the center of the circle, whereas ' $r$ ' reflects the concentration of phase values around the mean. Locomotor cycles in which the two VR activities were in complete alternation had a phase value of 0.5 . Those that were completely synchronous had phase values of 0 or 1 . Locomotor cycles with phase values from $0.25-0.75$ were defined as alternation and locomotor cycles with phase values $<0.25$ or $>0.75$ as synchronous. Rayleigh's test (Zar, 1974) was used to determine whether $r$ values reached statistical significance $(p<0.05)$.

Anatomical tracing. For the retrograde tracing experiments, spinal cords from neonatal transgenic mice ( $\mathrm{P} 0-\mathrm{P} 2)$ were isolated (see above). After dissection, crossing neurons (CNs) were labeled by applying crystals of Rhodamine dextran amine (3000 MW) to a transverse cut made between the L3 and L4 segment, as described previously (Stokke et al., 2002; Restrepo et al., 2009). To allow retrograde transport up to L2, preparations were incubated for $5-7 \mathrm{~h}$ in the dark in oxygenated normal Ringer's solution at room temperature. Thereafter, the spinal cords were immersed in $4 \%$ PFA in PBS overnight at $4^{\circ} \mathrm{C}$. The fixed tissues were then cryoprotected by incubation in $20 \%$ sucrose in PBS overnight at $4^{\circ} \mathrm{C}$ before embedding in Cryo-mount and rapidly frozen at $-80^{\circ} \mathrm{C}$.

For anterograde labeling of CST neurons, adult mice (10 weeks up to 3 months) were anesthetized with ketamine $(75 \mathrm{mg} / \mathrm{kg}) /$ medetomidine $(1$ $\mathrm{mg} / \mathrm{kg}$ ). Multiple injections ( $1.5 \mu \mathrm{l}$ total volume) of $10 \%$ biotin dextrane amine 10,000 (BDA) diluted in $0.01 \mathrm{M} \mathrm{PBS,} \mathrm{pH} 7.4$, were pressure injected into the forelimb and hindlimb area of the primary motor cortex (1.5 mm lateral to bregma, $800-900 \mu \mathrm{m}$ from the surface). After 14-21 $\mathrm{d}$, mice were transcardially perfused with $0.01 \mathrm{~m}$ PBS followed by $4 \%$ PFA in PBS, pH 7.4. The spinal cord and the brainstem were dissected out, postfixed in $4 \%$ PFA at $4^{\circ} \mathrm{C}$ overnight, and cryoprotected in $20 \%$ sucrose in PBS at $4^{\circ} \mathrm{C}$ overnight before embedded in Cryo-Mount and rapidly frozen in $-80^{\circ} \mathrm{C}$. 
A

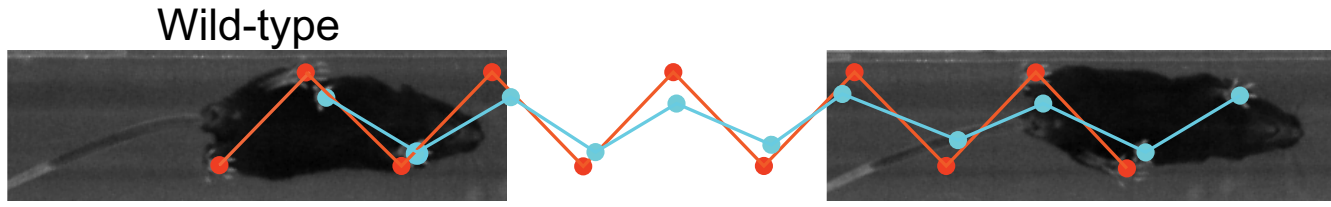

B
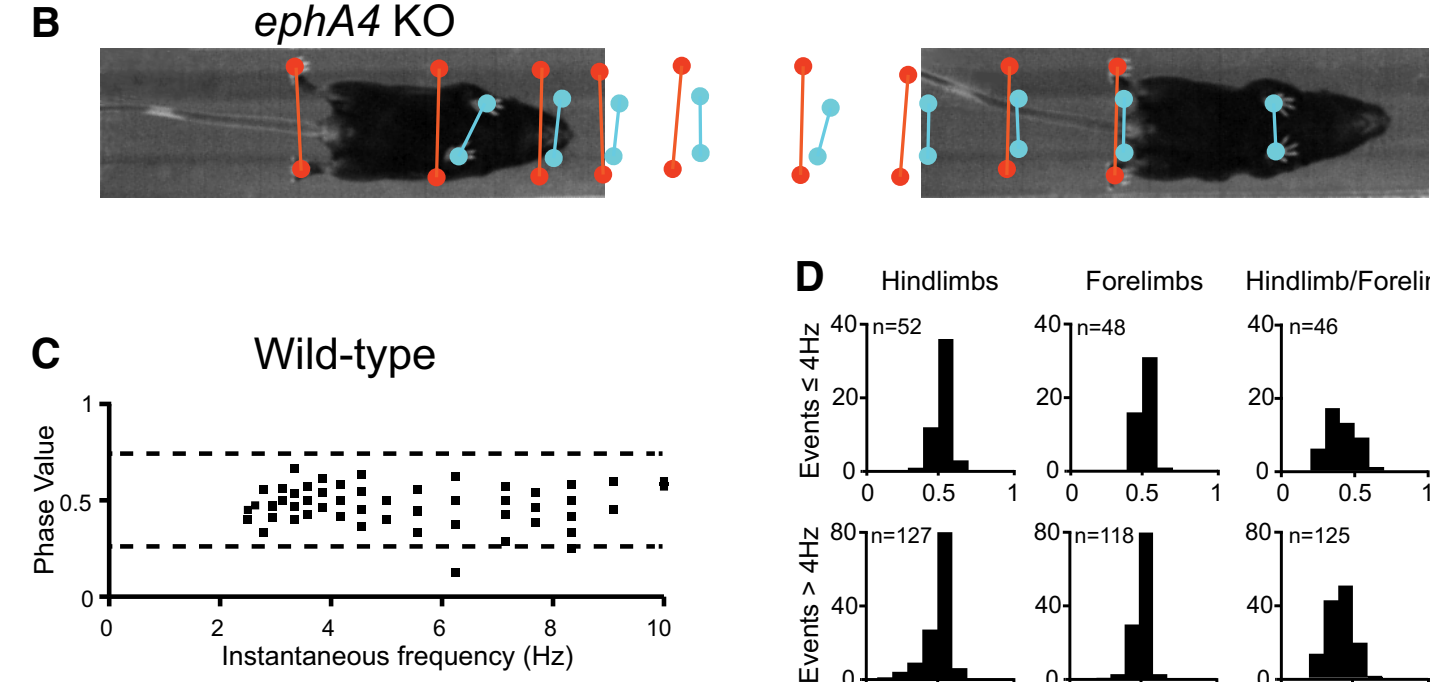

Forelimbs

Hindlimb/Forelimb
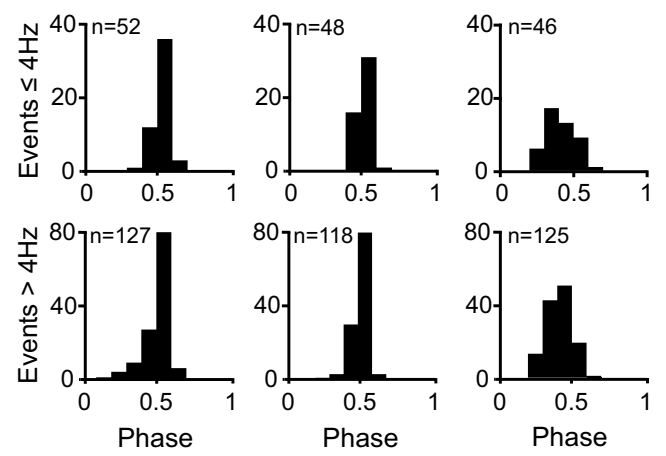

F Hindlimbs

Forelimbs Hindlimb/Forelimb

E

ephA4 KO
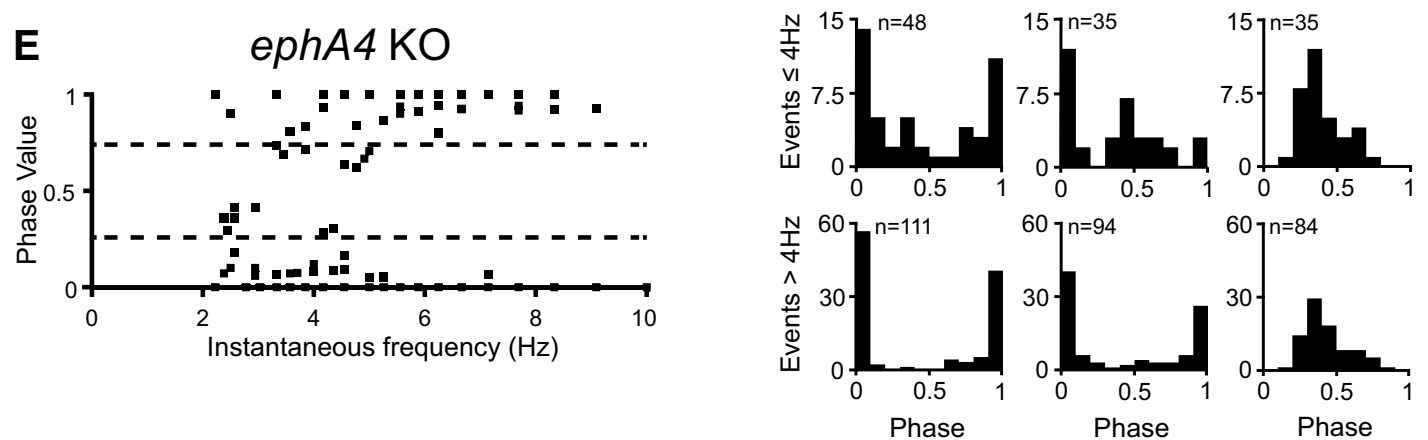

\section{H Hindlimbs}

Forelimbs
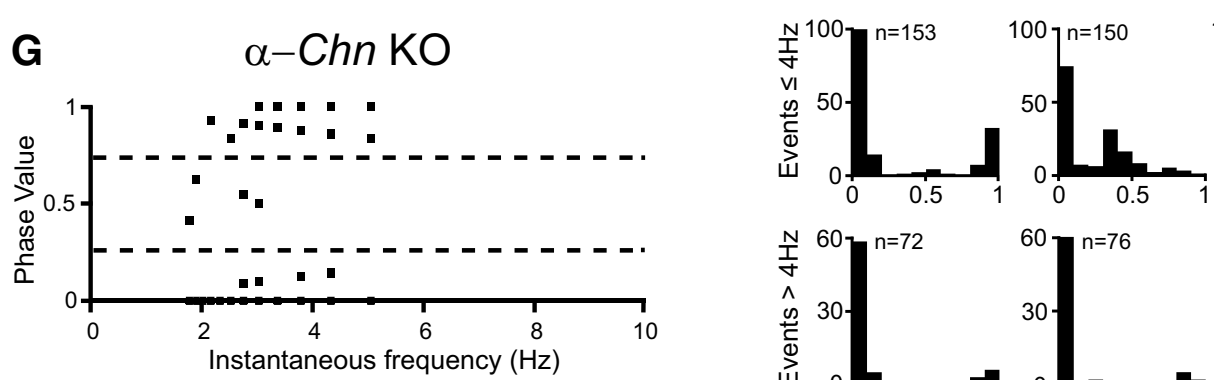

Hindlimb/Forelimb
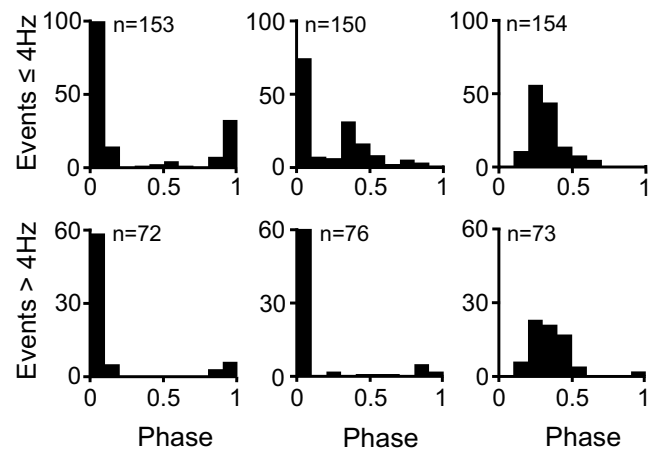

Figure 1. Locomotor pattern in freely moving wild-type, ephA4 K0 and $\alpha$-Chn KO mice. $\boldsymbol{A}, \boldsymbol{B}$, Illustration of locomotor pattern in wild-type $(\boldsymbol{A})$ and ephA4 KO (B) mice. Hindlimb movements are represented by red dots, and forelimb movements by light-blue dots. $\boldsymbol{C}, \boldsymbol{E}, \boldsymbol{G}$, Frequency-phase relationship of the locomotor gait in wild-type $(\boldsymbol{C} ; N=5)$, ephA4 KO (E; $N=7)$, and $\alpha$-Chn $\mathrm{KO}$ mice $(\boldsymbol{G} ; N=5)$. $\boldsymbol{D}, \boldsymbol{F}, \boldsymbol{H}$, Phase histograms showing the phase values between indicated pairs of limbs divided in low-frequency $(\leq 4 \mathrm{~Hz}$, top) and high-frequency ( $>4 \mathrm{~Hz}$, bottom) locomotion for wild-type $(\boldsymbol{D})$, ephA4 KO $(\boldsymbol{F})$, and $\alpha$-Chn KO $(\boldsymbol{H})$ mice. The same datasets were used in $\boldsymbol{C}$ and $\boldsymbol{D}, \boldsymbol{E}$ and $\boldsymbol{F}$, and $\boldsymbol{G}$ and $\boldsymbol{H} ; \boldsymbol{n}$ indicates total amount of analyzed steps. 
Immunohistochemistry. Serial transverse sections of the newborn lumbar spinal cord $(14-30 \mu \mathrm{m})$ and the adult cervical and lumbar spinal cord $(30 \mu \mathrm{m})$ were cut on a cryostat and mounted on Menzel-Glaser SuperFrost slides. All slides were kept at $-80^{\circ} \mathrm{C}$ until used for immunohistochemistry or in situ hybridization.

Sections were incubated overnight at $4^{\circ} \mathrm{C}$ with rabbit anti-Cre antibody (1:10,000; gift from G. Shutz) for the retrograde labeling experiments, mouse anti-NeuN, biotin-conjugated antibody (1:500, Millipore) for the dorsal funiculus experiments, or with a streptavidin, Alexa Flour 488 antibody (1:500; Invitrogen) for the anterograde labeling experiments. Secondary antibodies were obtained from Jackson ImmunoResearch and incubated for $1 \mathrm{~h}$ at room temperature. Slides were washed in 0.01 м PBS, mounted in glycerol/0.01 м PBS 1:1 and scanned on an LSM confocal microscope (Zeiss Microsystems) using an $\times 10$ or $\times 20$ objective. Multiple channels (when needed) were scanned sequentially to prevent fluorescence bleed through. Analyzed sections were collected $>50$ $\mu \mathrm{m}$ apart.

Antibody specificity. Antibody dilutions were determined by incrementing their concentration from that which yielded a negligible signal to that which yielded a reliable visualization of cell bodies. No signal was observed in control sections when the primary antibody was omitted. Rabbit anti-Cre (1:10,000; from G. Shutz) was used to visualize Vglut2 ${ }^{+}$ cells. The anti-Cre antibodies did not yield any staining in wild-type mouse tissues and have also been evaluated for specificity (Lundfald et al., 2007; Borgius et al., 2010).

In situ hybridization. Fluorescent in situ hybridization was performed using an EphrinB3 riboprobe (base pairs 411-1239) using an adopted protocol from Borgius et al. (2010) and visualized by TSA Fluorescein Plus Evaluation Kit (PerkinElmer). The sections were counterstained with mouse anti-NeuN (1:500, clone A60, MAB377; Millipore) followed by CY3 AP Donkey anti-mouse (1:500, 715-165-151; Jackson ImmunoResearch).

Cell counting. The quantifications for the retrograde labeling were performed on high-resolution images of individual sections using ImageJ. The total number of retrogradely labeled cells located in the entire transverse section of the spinal cord contralaterally to the side of injection was counted. For each putative retrogradely labeled neuronal profile, Cre expression was determined. Estimated colocalization events were normalized to the number of Vglut2-negative crossing neurons in the same sections $\left(\mathrm{CN}_{\text {Total }}-\mathrm{CN}_{\mathrm{Vglut} 2}{ }^{+}\right)$. Counts were performed in both Vglut2::Cre $(N=3)$ and Vglut2::Cre; ephA4 ${ }^{-/ \text {Flox }}(N=3)$ mice, in 8-10 sections from each animal. Sections were collected $>50 \mu \mathrm{m}$ apart and covered all of the L2 segment and the rostral part of the L3 segment.

Calculation of dorsal commissure, dorsal funiculus, and EphrinB3 expression. All measurements and surface area calculations were made manually on high-resolution images of individual sections using ImageJ. At least eight sections from each animal covering the upper lumbar segments (L1-L3) were analyzed. For the EphB3 barrier gap measurement, the difference in distance between the dorsal edge of the EphrinB3 barrier and the ventral edge of the dorsal funiculus was calculated and normalized to the distance between the central canal and the ventral edge of the dorsal funiculus. All data are expressed as means \pm SD. Statistical analysis was conducted using one-way ANOVA and Bonferroni's test with $p<0.05$ versus control.

\section{Results}

\section{Locomotion in mice lacking EphA4 signaling is dominated by} quadrupedal hopping

The locomotor pattern in the ephA4 and $\alpha$-Chn KO mice has been described in some detail before with analysis of a limited frequency domain of locomotion (Kullander et al., 2003; Akay et al., 2006; Iwasato et al., 2007). Here, we used a more elaborate locomotor analysis incorporating overground locomotion at frequencies between 2 and $10 \mathrm{~Hz}$. In this frequency range, freely running wild-type mice displayed walking (one limb in the air and alternation between left and right legs) or trotting gaits (diagonal limbs on the ground with intrasegmental alternation; phase values $\sim 0.5$; Talpalar et al. 2013; Fig. $1 A, C, D$, Movie 1).

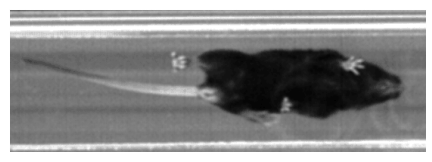

Movie 1. Alternating gait in wild-type mouse. Eight-week-old wild-type mouse running spontaneously on a runway. The run was captured at $100 \mathrm{frames} / \mathrm{s}$ and is shown at half-speed. At all frequencies of locomotion the mouse produces alternating gaits in both forelimbs and hindlimbs.

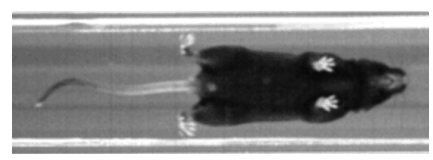

Movie 2. Quadrupedal hopping in ephA4 KO mouse. Eight-week-old ephA4 KO mouse running spontaneously on a runway. The run was captured at 100 frames/s and is shown at halfspeed. At all frequencies of locomotion the mouse shows a pronounced quadrupedal hopping with sequential synchronized lifting of the forelimbs followed by synchronized lifting of the hindlimbs.

During very high locomotor frequencies $(>10 \mathrm{~Hz})$ wild-type mice may gallop, moving their hindlimbs in synchrony (phase values $\sim 0$ or 1 ) and out of phase with the forelimbs that are alternating (C. Bellardita and O. Kiehn, unpublished observation). ephA4 KO mice when running freely on the runway predominantly showed a characteristic quadrupedal hopping gait, with synchronized lifting of the forelimbs followed by out of phase synchronized lifting of the hindlimbs (Fig. $1 B, E, F$, Movie 2). This quadrupedal hopping gait was dominant at all frequencies (Fig. 1E). However, occasionally during lower frequencies of locomotion $(<4 \mathrm{~Hz})$ the quadrupedal hopping gait was replaced with steps of alternation (Fig. $1 E, F$ ). The presence of alternation (phase values between 0.25 and 0.75 ) was significant (hindlimb: $p<0.0001$, forelimb: $p<0.0001$; Fisher's test) at low $(\leq 4 \mathrm{~Hz})$ frequencies of locomotion as compared with high $(>4 \mathrm{~Hz})$ frequencies of locomotion in the ephA4 KOs. There was no significant difference in the phase distributions between low and high locomotor frequencies in the wild-type mice. The transition between hopping and alternation occurred during active movements allowing a gait switch in a single locomotor step.

$\alpha-C h n \mathrm{KO}$ mice also showed a predominant quadrupedal hopping gait at all frequencies, with occasional alternation at the lower frequency range (Fig. $1 G, H$ ). No significant difference was seen between the distribution of hindlimb phase values between low and high frequencies for the $\alpha-C h n \mathrm{KO}$ mice, although there was significantly more alternation $(p<0.0001)$ between forelimbs at low frequencies of locomotion.

These experiments demonstrate that the quadrupedal hopping gait is dominating in the complete EphA4 signaling KOs. However, the ability to produce an alternating pattern of locomotion, particularly at lower locomotor frequencies, indicates that these EphA4 signaling KOs retain the neuronal circuitry responsible for alternation.

\section{Bilateral innervation by CST neurons in the spinal cord does not lead to a change in locomotor pattern}

The axonal expression of both the EphA4 receptors and its effector proteins together with the midline expression of EphrinB3 is needed for correct unilateral axonal branching of the CST neurons in the spinal cord (Dottori et al., 1998; Kullander et al., 2001; Yokoyama et al., 2001). When the EphA4 signaling is absent, CST axons cross aberrantly in the spinal cord, leading to a 
A
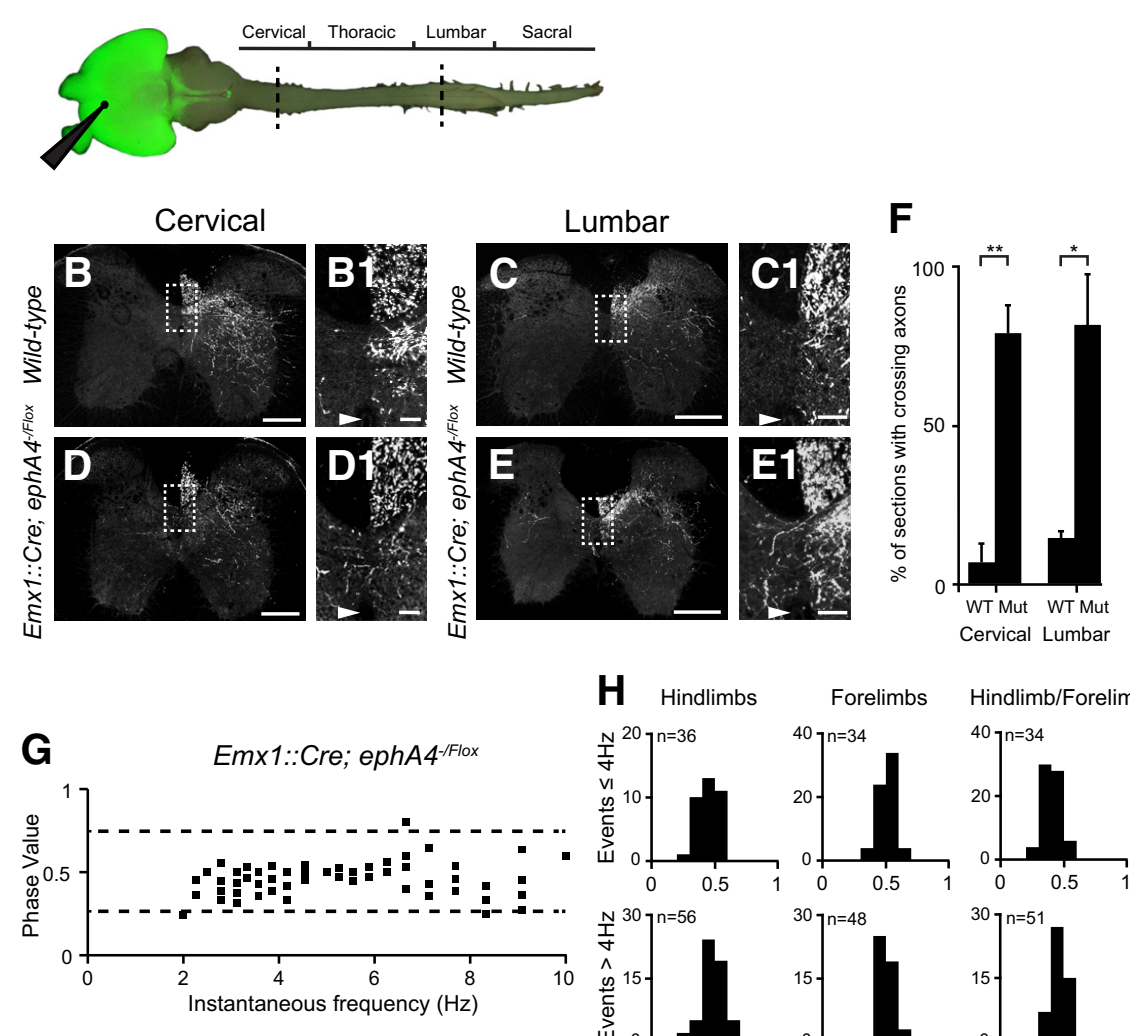

H Hindlimbs
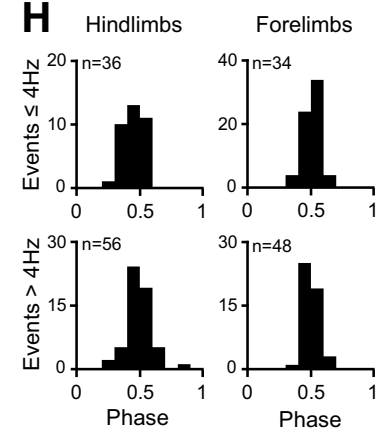

Hindlimb/Forelimb
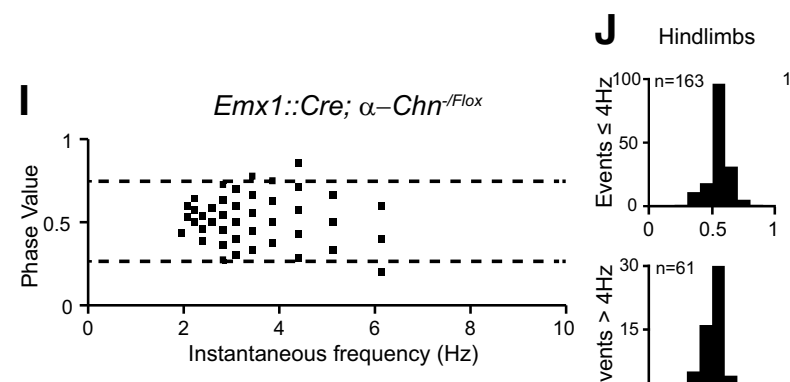

Forelimbs

Hindlimb/Forelimb
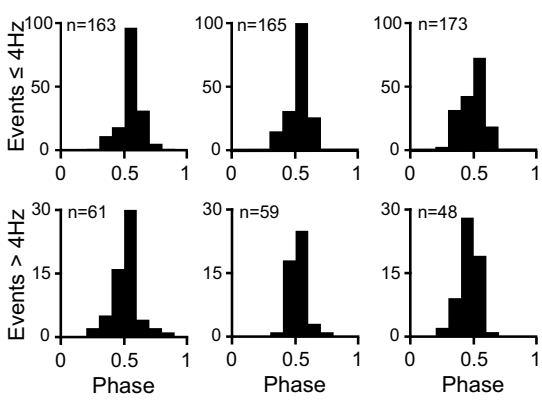

Figure 2. Cortical deletion of ephA4 or $\alpha$-Chn does not lead to a change in overground locomotor pattern. $A$, Expression of Emx1::Cre visualized by the expression of YFP in the Emx1::Cre; Rosa26::YFP mice. Recombination is exclusively detected in the cortex and no recombination is visible in the spinal cord. The injection site of biotinylated dextran amine (BDA) unilateral in motor cortex is shown by a black arrowhead. The location of the transverse sections showing CST branching in $\boldsymbol{B}-\boldsymbol{E}$ is marked with dashed lines. $\boldsymbol{B}-\boldsymbol{E}$, Transverse sections showing anterograde tracing with BDA of CST axons in wild-type and Emx $1::$ Cre; ephA4 ${ }^{-/ F l o x}$ mice in the cervical enlargement $(C 3-C 6 ; \boldsymbol{B}, \boldsymbol{D})$ and in the lumbar region ( $L 1-L 2 ; \boldsymbol{C}, \boldsymbol{E}) . \boldsymbol{B 1}-\boldsymbol{E 1}$, Enlargements of the boxed areas in $\boldsymbol{B}-\boldsymbol{E}$. The $C($ is indicated by a white arrowhead. $\boldsymbol{F}$, Quantification of sections with CST axons crossing the midline dorsal to the $C($ in wild-type and Emx1::Cre; ephA4 ${ }^{-/ F l o x}$ mice. Significantly more sections with crossing axons were detected in Emx1::Cre; EphA4 ${ }^{-/ \text {Flox }}$ mice (Mut) compared with wild-type mice (WT), both in the cervical enlargement, C3-C6 (78.8 $\pm 6.5 \%$ against $6.7 \pm 5.9 \%, p<0.01)$ and in the lumbar area, $\mathrm{L} 1-\mathrm{L} 2(81.5 \pm 15.8 \%$ against $7.1 \pm 2.0 \% p<0.05)$, (mean $\pm \mathrm{SD} ; N=2$ for each genotype, $30-50$ sections per area and animal). Scale bars: $\boldsymbol{B}-\boldsymbol{E}, 300 \mu \mathrm{m} ; \boldsymbol{B} \mathbf{B}-\mathbf{E 1}, 50 \mu \mathrm{m} . \boldsymbol{G}, \boldsymbol{I}$, Frequency-phase relationship of the locomotor gait in Emx1::Cre; ephA4 ${ }^{-/ F l o x}(N=6)$ and $E m \times 1:: C r e ; \alpha-C h n^{-/ F l o x}(N=5)$ mice. $\boldsymbol{H}, \boldsymbol{J}$, Phase histograms showing the phase values between indicated pairs of limbs divided in low-frequency ( $\leq 4 \mathrm{~Hz}$, top) and high-frequency $(>4 \mathrm{~Hz}$, bottom)

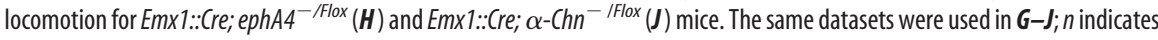
total amount of analyzed steps.

bilateral instead of predominantly contralateral innervation of the spinal cord.

To evaluate if the perturbed branching of CST axons alone might have an influence on the overground locomotor pattern

observed in the complete EphA4 signaling KOs, we first eliminated EphA4 signaling specifically from the CST axons by a restricted deletion of the ephA4 gene from cortical neurons without affecting the EphA4 expression in the spinal cord. This was obtained by partnering the forebrainrestricted Emx1::Cre; ephA $4^{-/+}$mice (Fig. $2 A$ ) with $e p h A 4^{F l o x}$ mice to generate conditional Emx1::Cre; ephA4 ${ }^{-/ \text {Flox }}$ mice. In the Emx1::Cre; ephA4 ${ }^{-/ F l o x}$ mice there was an increased midline re-crossing of CST axons dorsal to the central canal (CC), both in the cervical enlargement (C3-C6) and in the lumbar spinal cord (L1-L2; Fig. $2 B-F)$, confirming that the recombination was efficient. When running spontaneously on the runway the Emx1::Cre; ephA $4^{-I F l o x}$ mice showed strict alternating gait patterns at all frequencies of locomotion (Fig. 2G,H) completely resembling wild-type mice gait patterns (Movie 3). Emxl::Cre; $\alpha$-Chn ${ }^{- \text {IFlox }}$ mice also showed a strict alternating gait at all frequencies of locomotion (Fig. 2I,J). Neither Emxl::Cre; ephA4 ${ }^{-/ \text {Flox }}$ nor Emx1::Cre; $\alpha-\mathrm{Chn}^{-/ \text {Flox }}$ mice showed any significant difference in phase value distributions of either hindlimbs or forelimbs at low compared with high frequencies of locomotion.

Therefore, despite the large increase of CST axons aberrantly re-crossing the midline, both in the cervical enlargement and in the lumbar spinal cord, the bilateral innervation of CST axons is insufficient to mediate a change in gait during overground locomotion regardless of locomotor frequency.

Deletion of EphA4 signaling in spinal neurons converts the alternating gait into a hopping locomotor gait

We next evaluated the consequence of the reverse manipulation: removal of the EphA4 signaling regionally in the spinal cord without affecting supraspinal areas. For this purpose, we partnered the spinal cord-restricted Hoxb8::Cre; ephA4 $4^{-/+}$ transgenic mice with homozygous EphA4 ${ }^{\text {Flox }}$ mice. The homeodomain protein Hoxb8 is expressed exclusively from cervical segment 4 caudally in the spinal cord (Fig. $3 A$ ). Hoxb8::Cre; ephA4 ${ }^{-/ F l o x}$ mice running spontaneously on the runway displayed a hopping hindlimb gait at all frequencies of locomotion (Fig. $3 B$, Movie 4) with occasional events of alternation at frequencies around and $<4 \mathrm{~Hz}$ (Fig. 3 B, C, left). These events with alternation were usually seen in the beginning or at the end of a run where the frequency of locomotion is low and reflected in the significant increase of alternation of the 
hindlimbs at low compared with high frequencies of locomotion $(p<0.0001)$.

The coordination between the left and right forelimbs varied between alternation and periods of synchrony with more alternation at lower (Fig. 3C, middle; $p<0.001$ ) compared with higher frequencies of locomotion.

These experiments demonstrate that a complete elimination of EphA4 signaling from all neurons from C4 caudally in the spinal cord is sufficient to generate a synchronous locomotor activity in the hindlimbs, remarkably similar to the hindlimb locomotor pattern found in the complete EphA4 signaling KOs.

The locomotor gait is dominated by a hopping phenotype when EphA4 signaling is deleted from all Vglut $2^{+}$ neurons

The experiments with selective elimination of EphA4 signaling from spinal or supraspinal neurons point to a spinal identity of the neurons responsible for the hopping phenotype, but they do not reveal the molecular identity of these neurons. It has previously been shown that an increase in synaptic inhibition in the isolated spinal cord preparation from ephA4 KO mice can revert the synchronous locomotor-like activity back to alternation (Kullander et al., 2003), indicating that a spinal cord-specific lack of EphA4 signaling in excitatory neurons may cause the hopping phenotype. To functionally test this hypothesis we selectively removed EphA4 signaling from glutamatergic neurons using Vglut2::Cre mice, since Vglut2 is the vesicular glutamate transporter found in most interneurons in the ventral spinal cord (Borgius et al. 2010; Talpalar and Kiehn, 2010). The EphA4 signaling was eliminated in two different ways: the Vglut2::Cre; ephA $4^{-/+}$mice were partnered with ephA4 $4^{\text {Flox }}$ mice to generate Vglut2::Cre; ephA4 $4^{-/ \text {Flox }}$ mice and to further confirm the importance of EphA4 signaling in excitatory neurons we also partnered Vglut2::Cre; $\alpha$-Chn ${ }^{-/+}$mice with $\alpha$-Chn ${ }^{\text {Flox }}$ mice to generate Vglut2::Cre; $\alpha-C h n^{-/ F l o x}$ mice.

When running freely on the runway, the Vglut2::Cre; ephA4 ${ }^{-/ \text {Flox }}$ mice displayed a quadrupedal hopping gait at all frequencies of locomotion with occasional events of alternation (Fig. $4 A, B$, Movie 5 ) at frequencies below and around $4 \mathrm{~Hz}$. These events with alternation occurred infrequently, and were usually seen in the beginning or at the end of a run. Also the Vglut2::Cre; $\alpha$-Chn ${ }^{-/ \text {Flox }}$ mice displayed a quadrupedal hopping gait independent of the speed of locomotion when running freely on the runway (Fig. $4 C, D$ ) although a higher degree of alternation at frequencies below and around 4 $\mathrm{Hz}$ was observed compared with the Vglut2::Cre; ephA4 $4^{-/ \text {Flox }}$

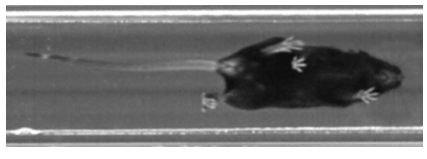

Movie 3. Alternating gait in Emx1:Cre; ephA4 ${ }^{-/ F l o x}$ mouse. Eight-week-old Emx1:Cre; ephA4 $4^{-/ F l o x}$ mouse running spontaneously on a runway. The run was captured at $100 \mathrm{frames} / \mathrm{s}$ and is shown at half-speed. At all frequencies of locomotion, the mouse produces alternating gaits in both forelimbs and hindlimbs.

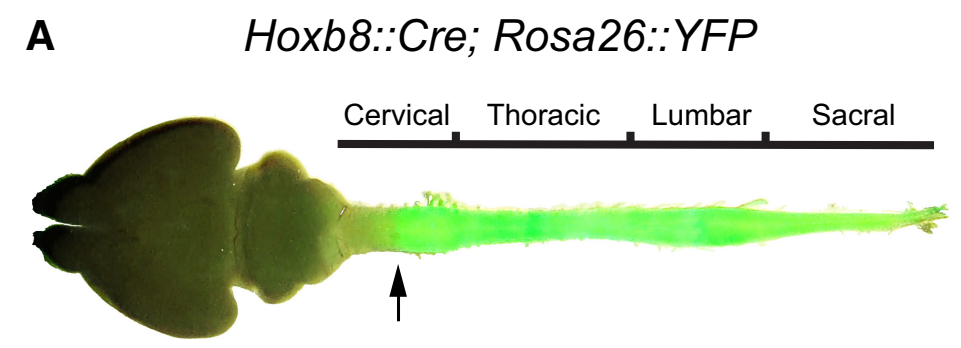

B Hoxb8::Cre; ephA4-/Flox
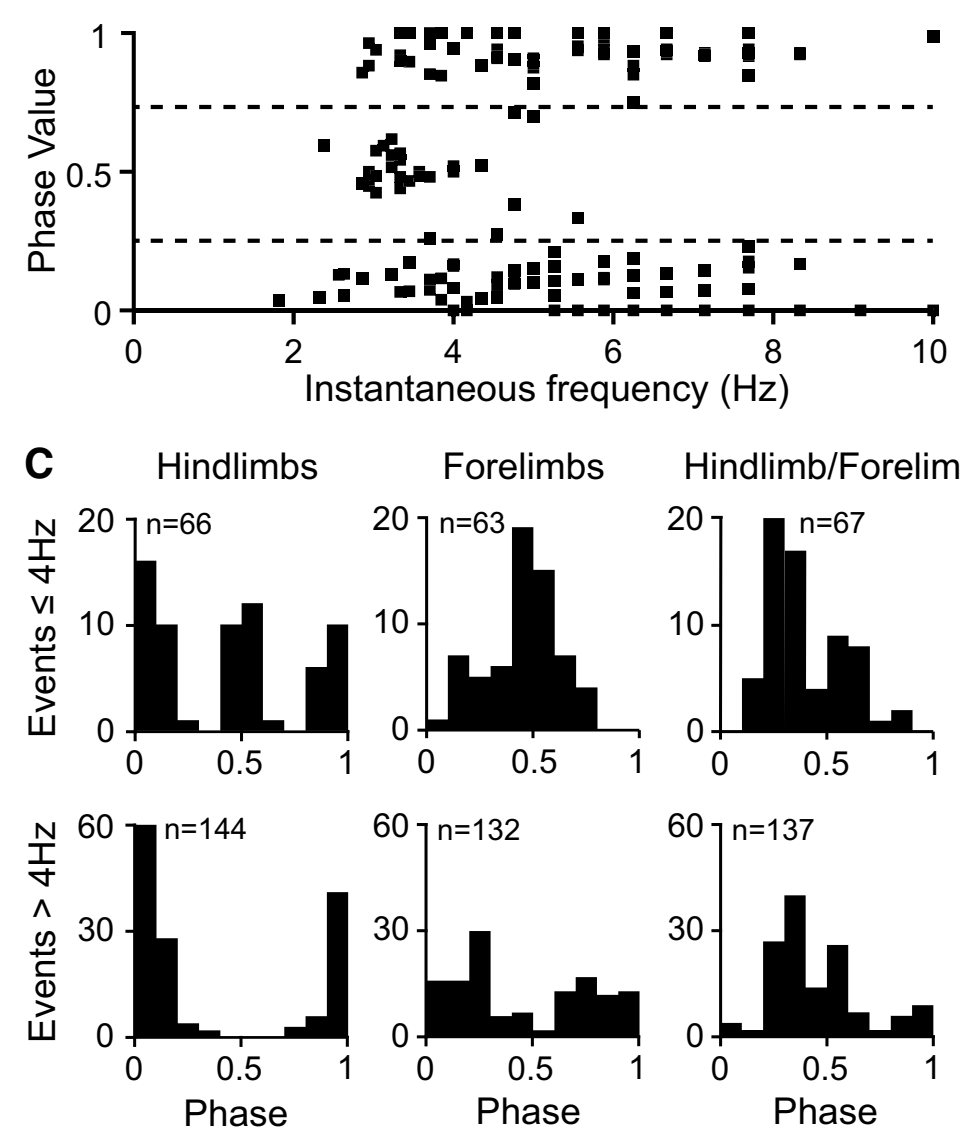

Figure 3. Selective deletion of EphA4 from the spinal cord leads to a hopping hindlimb gait. $\boldsymbol{A}$, Expression of Hoxb8::Cre visualized by the expression of YFP in the Hoxb8::Cre; Rosa26::YFP mice. Recombination is exclusively detected in the spinal cord with a rostrocaudal limit at the fourth cervical root (C4) indicated by an arrow. $\boldsymbol{B}$, Frequency-phase relationship of the locomotor gait in Hoxb8::Cre; ephA4 ${ }^{-/ F l o x}(N=5)$ mice. C, Phase histograms showing the phase values between indicated pairs of limbs divided in low-frequency ( $\leq 4 \mathrm{~Hz}$, top) and high-frequency ( $>4 \mathrm{~Hz}$, bottom) locomotion for Hoxb8::Cre; ephA4 ${ }^{-/ \text {Flox }}$ mice using the same dataset as shown in $\boldsymbol{B} ; n$ indicates total amount of analyzed steps.

mice. Both Vglut2::Cre; ephA4 ${ }^{-/ \text {Flox }}$ and Vglut2::Cre; $\alpha-C h n^{-/ F l o x}$ mice showed generally a significant increase of alternation at low compared with high frequencies of locomotion (Vglut2::Cre; ephA4 $4^{-/ \text {Flox }}$, hindlimbs: $p<0.0001$, forelimbs: $p<0.001$; 


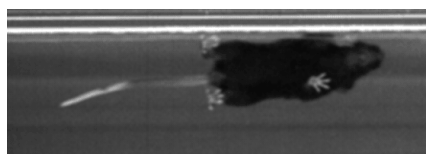

Movie 4. Hindlimb hopping in Hoxb8::Cre; ephA4 $4^{-/ F l o x}$ mouse. Five-week-old Hoxb8::Cre;ephA4 $4^{-/ F l o x}$ mouse running spontaneously on a runway. The run was captured at $100 \mathrm{frames} / \mathrm{s}$ and is shown at half-speed. At all frequencies of locomotion the mouse displays hindlimb hopping while the forelimb is mostly alternating, reflecting the rostrocaudal gradient of Hoxb8 expression in the spinal cord.

roots accompanied by alternating flexor (L2)-extensor (L5) activity in preparations from both Vglut2::Cre; ephA4 ${ }^{-/ \text {Flox }}$ and Vglut2::Cre; $\alpha-C h n^{-/ F l o x}$ mice. Although the synchronous activity was the most prominent induced locomotor phenotype in preparations from both Vglut2-specific EphA4 signaling KOs, sequences with relatively normal alternating locomotor-like activity were also generated. In many of the preparations that exhibited a synchronous activity, the phase values between leftright bursts were not completely stable and we observed periods of drifting between synchronous activity and alternation as seen in the circular plots (Fig. $5 B, C, G, H$ ).

For the Vglut2::Cre; ephA4 $4^{-/ \text {Flox }}$ preparations, the frequency of the drug-evoked locomotor-like activity ranged between 0.30 and $0.60 \mathrm{~Hz}$ (mean value \pm SD: $0.45 \pm$ $0.06 \mathrm{~Hz}, N=9)$. To evoke locomotor-like activity with higher frequency we used electrical stimulation of the cervical spinal cord (Fig. 5D). This stimulation caused a stable hopping phenotype for the duration of the stimulation in all preparations tested $(N=6)$ and the frequency of locomotor-like activity was between 0.55 and $0.75 \mathrm{~Hz}$ (mean value $\pm \mathrm{SD}: 0.63 \pm$ $0.06 \mathrm{~Hz}$ ). For the Vglut2::Cre; $\alpha$-Chn ${ }^{-/ \text {Flox }}$ mice, the frequency of the drug-evoked locomotor-like activity evoked by superfusion of locomotor drugs with concentrations typically used in previous studies (NMDA, 5.0-7.5 $\mu \mathrm{M}$; 5-HT, $5 \mu \mathrm{M}$ ) ranged from 0.37 to $1.42 \mathrm{~Hz}$ (mean value $\pm \mathrm{SD}$ : $0.56 \pm 0.26 \mathrm{~Hz}, N=11)$. To examine whether the spinal locomotor network of Vglut2::Cre; $\alpha$-Chn ${ }^{-/ \text {Flox }}$ mice generates synchronous left-right pattern at higher locomotor frequencies, we superfused the in vitro spinal cord preparation with higher concentrations of NMDA (10-30 $\mu \mathrm{M}, N=18)$ to increase the locomotor

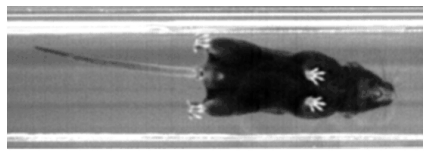

Movie 5. Quadrupedal hopping in Vglut2::Cre; ephA4 ${ }^{-/ F l o x}$ mouse. Eight-week-old Vglut2:::Cre; ephA4 ${ }^{- \text {Flox }}$ mouse running spontaneously on a runway. The run was captured at 100 frames/s and is shown at half-speed. At all frequencies of locomotion the mouse shows a pronounced quadrupedal hopping with sequential synchronized lifting of the forelimbs followed by synchronized lifting of the hindlimbs.

Vglut2::Cre; $\alpha$-Chn ${ }^{-/ \text {Flox }}$, hindlimbs: $p<0.001$, forelimbs: $p>$ $0.05)$.

To confirm the spinal origin of the excitatory neurons generating the hopping phenotype we performed locomotor experiments using neonatal isolated spinal cords from both Vglut2::Cre; ephA4 $4^{-/ \text {Flox }}$ (Fig. 5A-D) and Vglut2::Cre; $\alpha-C h n^{-/ \text {Flox }}$ mice (Fig. $5 F-\mathrm{I})$. Locomotor-like activity was induced in isolated spinal cord preparations by adding neuroactive drugs, NMDA and 5 -HT, to the superfusion. This combination of neuroactive substances induced a "hopping" locomotor-like activity seen as synchronous activity in flexor-dominated left-right ventral L2 roots and in the extensor-dominated left-right ventral L5 drive (Talpalar and Kiehn 2010). The mean frequency of the induced rhythm was $0.60 \pm 0.24 \mathrm{~Hz}$, at $5.0-10.0 \mu \mathrm{MNMDA}(n=31)$ and significantly increased at 12.5-20.0 $\mu \mathrm{M} \mathrm{NMDA}(0.85 \pm 0.22 \mathrm{~Hz}, n=$ $30, p<0.01)$ and 22.5-30.0 $\mu \mathrm{M}$ NMDA $(1.06 \pm 0.25 \mathrm{~Hz}, n=30$, $p<0.01)$. The percentage of the preparations that showed significant synchronous activity between the left and right side of the cord increased with the higher frequencies induced by the higher concentrations of NMDA (Fig. 5I, left; 2730 cycles, $n=91$ ). Similarly, in wild-type $\left(\alpha-C h n^{-/ F l o x}\right)$ animals $(N=11)$, rhythmic activity with higher frequencies could be evoked by increasing the concentration of NMDA $(0.67 \pm 0.17 \mathrm{~Hz}$ at $5.0-10.0 \mu \mathrm{M}, n=22$; $0.90 \pm 0.19 \mathrm{~Hz}$ at $12.5-20.0 \mu \mathrm{M} n=16 ; 22.5-30.0 \mu \mathrm{M}$ at $1.24 \pm$ $0.19 \mathrm{~Hz}, n=25)$. However, the increase in frequency did not lead to synchronous left-right coordination (Fig. 5I, right; $1890 \mathrm{cy}$ cles, $n=63$ ).

In summary, the evoked locomotor-like activity in isolated spinal cords from the Vglut2-specific EphA4 signaling KOs resembled the locomotor phenotype observed in vivo with an overall hopping phenotype, especially at high locomotor frequencies, but with preserved alternating circuits.

These experiments demonstrate that a selective elimination of EphA4 signaling from glutamatergic Vglut $2{ }^{+}$neurons generates 
A

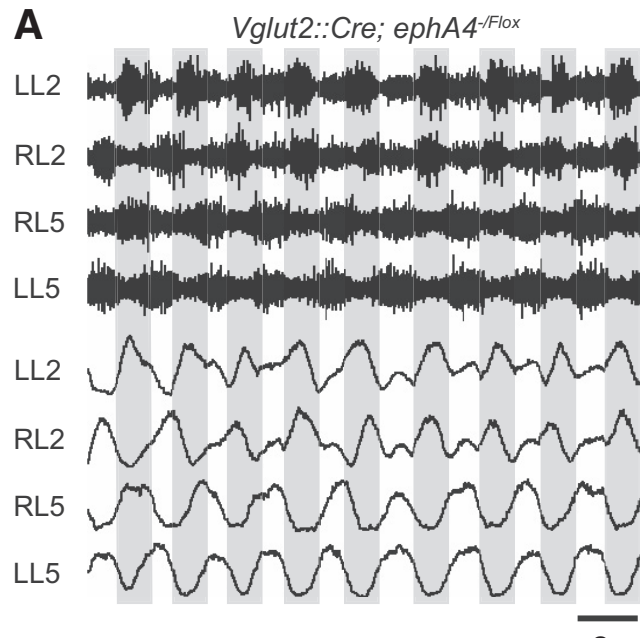

$\mathbf{F}$

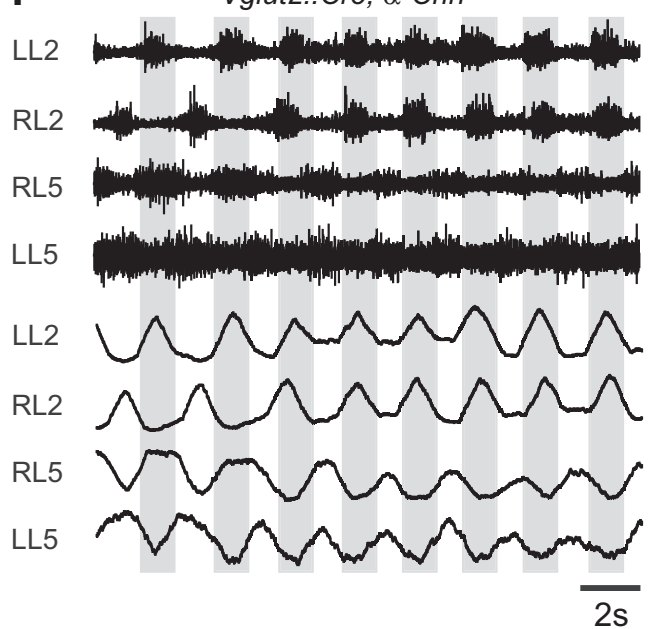

B

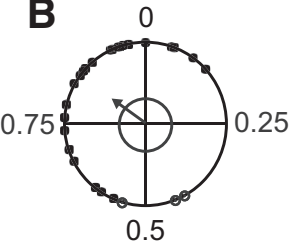

LL2 vs RL2

C

0.75

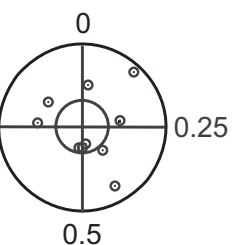

LL2 vs RL2

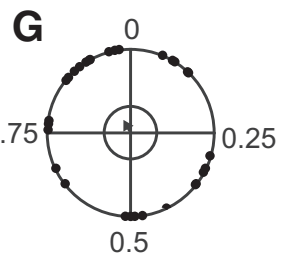

LL2 vs RL2

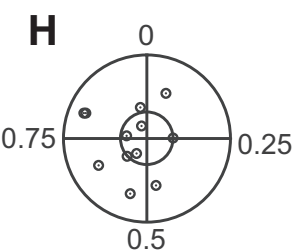

LL2 vs RL2
D Vglut2::Cre; ephA4-/Flox

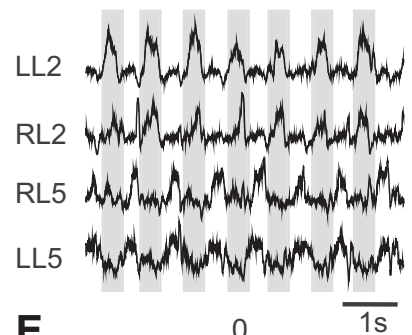

E

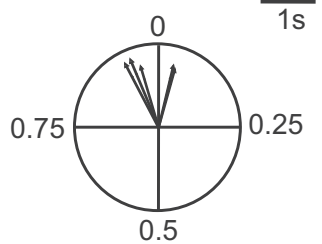

LL2 vs RL2
Vglut2::Cre; $\alpha-C h n^{-/ / 10 x} \quad$ Control

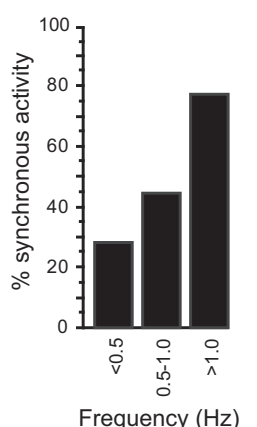

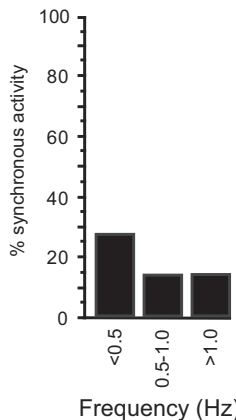

Figure 5. Locomotor-like activity in isolated spinal cord preparations. A, Raw VR recordings from a Vglut2::Cre; ephA4-/Flox mutant during drug-evoked locomotor-like activity showing left (L) and right (R) lumbar (L) 2 and 5 VRs (upper traces), with corresponding smoothed rectified traces below. B, Circular plot showing left-right (LL2-RL2) coordination obtained from the preparation shown in A. C, Circular plot showing left-right (LL2-RL2) coordination during drug-evoked locomotor-like activity from all Vglut2:: (re; ephA4-/Flox mutants examined ( $N=9$ ). D, Stimulation of descending fibers in a Vglut2:::Cre; ephA4-/Flox mutant. $E$, Circular plot showing left-right (LL2-RL2) coordination during descending stimulation from all Vglut2::: Cre; ephA4 $^{-/ F l o x}$ mutants examined $\left(N=6\right.$, left). $\boldsymbol{F}$, Raw VR recordings from a V glut2:: Cre; $\alpha$-Chn ${ }^{-/ F l o x}$ mutant during drug-evoked locomotor-like activity with corresponding smoothed rectified traces below. G, Circular plot showing left-right (LL2-RL2) coordination obtained from the preparation shown in $\boldsymbol{F}$. $\boldsymbol{H}$, Circular plot showing left-right (LL2-RL2) coordination during drug-evoked locomotor-like activity from all

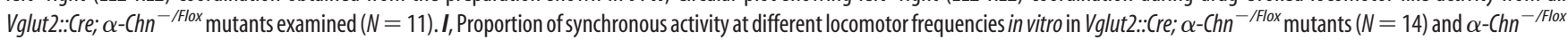
(control, $N=12$ ).

a hopping locomotor pattern that can be ascribed to a reconfiguration of the glutamatergic spinal locomotor network.

\section{Disruption of EphA4 signaling in glutamatergic neurons} leads to increase in midline crossing of Vglut ${ }^{+}$neurons As the hopping phenotype in the complete ephA4 KO was linked to an increased aberrant midline crossing of fibers in the spinal cord (Kullander et al., 2003), we investigated if the Vglut2specific deletion of EphA4 signaling led to an increase in midline crossing specifically of excitatory Vglut $2^{+}$neurons.

Crossing neurons and fibers in Vglut2::Cre; ephA4 ${ }^{-/ \text {Flox }}$ and Vglut2::Cre control mice were labeled by applying dextran tracers to one side of the cord in L3/L4 to reveal aberrant crossing and back-labeled neurons in L2. These experiments revealed an overall increase in axons crossing the midline in the Vglut2::Cre; ephA4 ${ }^{-/ F l o x}$ mice as compared with the Vglut2::Cre control mice (Fig. 6B,C).

The proportion of crossing Vglut $2^{+}$neurons, identified by both dextran labeling and Cre expression, in relation to the number of Vglut2-negative crossing neurons (CNs), was significantly increased in the Vglut2::Cre; ephA4 $4^{-/ \text {Flox }}$ mice $(0.86 \pm 0.26 ; N=$ $3)$ as compared with the Vglut2::Cre control mice $(0.47 \pm 0.23$; $N=3, p=<0.001$; Fig. $6 D--G)$. This increase in Vglut $2^{+}$overcrossing was reflected in a significant increase in the total number of crossing neurons in relation to the number of Vglut2-negative CNs in the Vglut2::Cre; ephA4 $4^{- \text {IFlox }}$ mice $(1.86 \pm 0.26 ; N=3$ ) compared with the Vglut $2::$ Cre control mice $(1.47 \pm 0.23 ; N=3$, $p=<0.001)$.

These findings link the hopping phenotype to an increased midline crossing of Vglut2 ${ }^{+}$neurons.

Altered dorsal funiculus anatomy is correlated with a dorsal gap in the EphrinB3 midline barrier

In both the ephA4 and $\alpha$-Chn KO mice there is a change in the shape of the dorsal funiculus (DF) and an increased distance between the CC and the ventral border of the DF (Dottori et al., 1998; Coonan et al., 2001; Kullander et al., 2001; Iwasato et al., 2007). It was therefore of interest to determine whether 
A

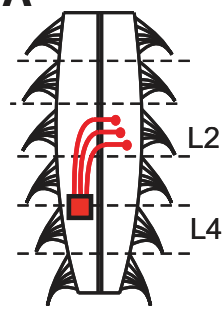

Rhodamine-D
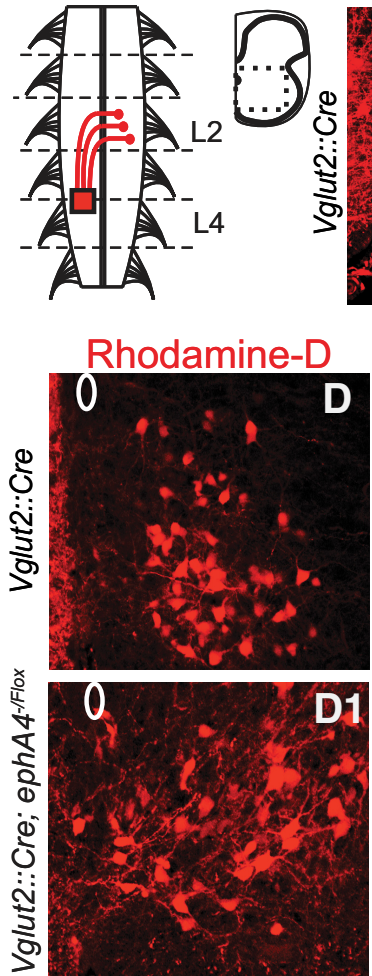

\section{Rhodamine-D}

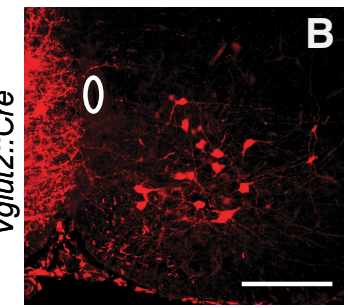

Cre
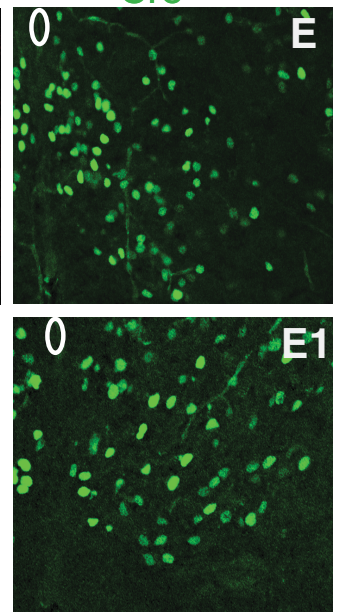

Rhodamine-D

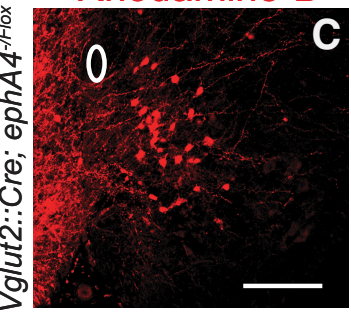

Rhodamine-D/Cre
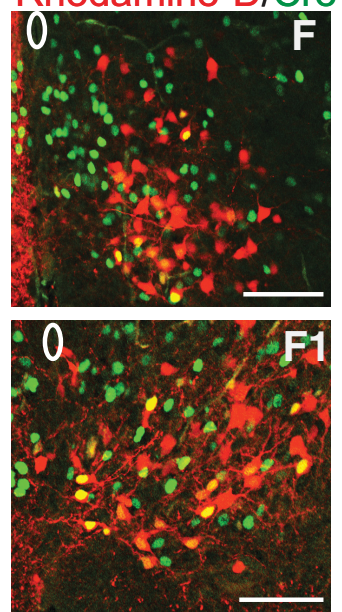

G

- Vglut2::Cre

- Vglut2::Cre; ephA4-/Flox

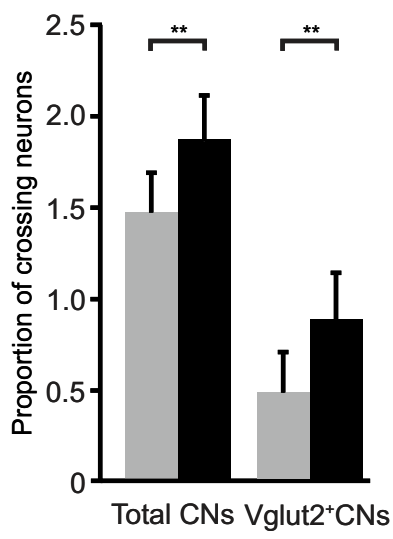

Figure 6. Increased midline crossing of Vglut2-positive neurons in the ventral spinal cord. $\boldsymbol{A}$, Schematic picture illustrating the site for the rhodamine tracer application (red box). $\boldsymbol{B}, \boldsymbol{C}$, Transverse sections of the $\mathrm{L} 2$ segment showing rhodamine dextran back-labeling of neurons and axons in Vglut2::Cre (B) or Vglut2::Cre; ephA4-/Flox mice (C). D-F1, Transverse sections from the L2 segment showing increase in rhodamine dextran back-labeled neurons (red) that are Vglut2-positive (green), identified by Cre expression, in the Vglut2::Cre;ephA4-/Flox KOs compared with the Vglut2::Cre controls. G, Quantification of retrograde-labeled CNs (left) labeled by rhodamine dextran in Vglut2::Cre compared with Vglut2::Cre;ephA4-/Flox mice in, as well as the proportion of retrogradelabeled CNs that are Vglut2 positive (right) in Vglut2::Cre compared with Vglut2::Cre; ephA4 ${ }^{-/ \text {Flox }}$ mice; all values normalized to Vglut2-negative neurons ( $N=3$ in each group; mean \pm SD, ${ }^{* *} p=$ $<0.001)$. The CC is indicated by a white circle. Scale bars: $\boldsymbol{B}, \boldsymbol{C}, 200 \mu \mathrm{m} ; \boldsymbol{D}-\boldsymbol{F}, \mathbf{D 1}-\boldsymbol{F 1}, 100 \mu \mathrm{m}$.

this was also the case in the different conditional KOs used in this study.

The anatomy of the DF was examined in the lumbar spinal cord of newborn mice. As previously described, the ephA4 KO mice had a significantly wider gap between the CC and the ventral edge of the DF relative to the total distance of the spinal cord dorsal to the CC $(0.65 \pm 0.004 ; N=2)$ compared with wild-type mice $(0.43 \pm 0.053$; $N=3$, $p<0.0001$; Fig. 7 A-C). No significant change in this distance was seen in the Emx1::Cre; ephA $4^{-/ \text {Flox }}$ mice $(0.35 \pm 0.029 ; N=3)$. In contrast, both the Hoxb8::Cre; ephA4 ${ }^{-/ \text {Flox }} \mathrm{KO}(0.73 \pm 0.02 ; N=$ $2, p<0.0001)$ and the Vglut2::Cre; ephA4 ${ }^{-/ \text {Flox }} \mathrm{KO}(0.74, \pm 0.0001$; $N=3, p<0.0001$ ), had a significant wider gap between the CC and the ventral edge of the DF resembling what was seen in the complete ephA4 KO mice. Similar results were obtained in the $\alpha$-Chn mutants where both the $\alpha$-Chn KO mice $(0.72 \pm 0.03, N=3)$ and the Vglut2::Cre; $\alpha$-Chn ${ }^{-/ \text {Flox }}$ mice $(0.68 \pm 0.03, N=3)$ had a wider gap compared with the control $\left(\alpha-C h n^{-/ F l o x}\right)$ mice $(0.45 \pm 0.07, N=3$, $p<0.0001)$. No significant difference was seen in the Emx1::Cre; $\alpha$-Chn ${ }^{- \text {Flox }}$ mice $(0.44 \pm 0.06, N=3)$.

The increased gap between the $\mathrm{CC}$ and the ventral edge of the DF seen in the ephA4 KO, Hoxb8::Cre; ephA4 ${ }^{-/ F l o x}$, and Vglut2::Cre; ephA $4^{-/ \text {Flox }}$ mice was accompanied by a change in shape of the DF where the DF was wider and the distance from the ventral edge of the DF to the surface of the spinal cord shorter compared with wild-type mice (Fig. 7A). However, the total areas of the DF in the different KOs used in this study were not significantly different (Fig. 7D). How this clear change in shape of the DF between the wild-type and the different conditional KO mice affects the ascending or descending fibers in the DF is not yet clear.
In wild-type mice, EphrinB3 forms a midline barrier throughout the dorsal and ventral commissure preventing EphA $4^{+}$axons from crossing the midline (Kullander et al., 2001). The increased gap between the CC and the ventral edge of the DF may be accompanied by a gap in the EphrinB3 expression, and to examine this we performed in situ hybridization using the lumbar spinal cord of newborn mice from the different conditional $\mathrm{KO}$ mice used in this study. In wild-type animals the EphrinB3 barrier stretches from the CC to the ventral edge of the DF (Fig. $7 E$ ). In contrast, in the conditional KO mice (Hoxb8::Cre; ephA4 ${ }^{-/ \text {Flox }}$ and Vglut2::Cre; ephA4 ${ }^{-/ \text {Flox }}$ ) and in the complete ephA4 KO with enlarged dorsal commissure, the EphrinB3 expression was lacking in the most dorsal part of the dorsal commissure (Fig. 7E,F). There was no gap in the EphrinB3 expression in the Emx1::Cre; ephA4 $4^{-/ \text {Flox }}$ mice (Fig. 7 E, F). None of the different KOs showed any disturbed midline structure or lack of EphrinB3 expression ventral to the CC.

In summary, when EphA4 signaling is affected in the spinal cord there is a widening of the distance between the CC and the ventral edge of the DF accompanied by a gap in the EphrinB3 barrier most dorsally in the dorsal commissure, as also reported when EphA4 is deleted from entire populations of dorsally derived spinal cord neurons (Paixão et al., 2013).

Aberrant midline crossing ventral to the central canal generates the hopping phenotype in the Vglut2-specific EphA4 signaling KO

The perturbed EphrinB3 barrier dorsal to the CC may lead to overcrossing of axons that is not directly related to elimination of the 
EphA4 signaling in specific cell populations. In a previous study of the complete ephA4 KO we have shown that the fibers responsible for generating the hopping phenotype cross in the ventral commissure (Restrepo et al., 2011). We confirmed this finding in the present study using Vglut2-specific EphA4 signaling KOs. Isolated spinal cords from Vglut2::Cre; $\alpha$-Chn ${ }^{-/ \text {Flox }}$ or $\alpha-C h n^{-/ F l o x}$ control mice were surgically cut from the dorsal surface of the spinal cord to the CC, covering the complete dorsal commissure. The same concentration of NMDA and 5-HT was used for inducing locomotor-like activity in the isolated spinal cord preparations before and after the lesion. In spinal cords isolated from Vglut2::Cre; $\alpha$-Chn ${ }^{-/ F l o x}$ we found that the hopping phenotype with segmental leftright synchrony was preserved after lesion ( $n=6$; Fig. 8). The alternating pattern of locomotion seen in the $\alpha$-Chn ${ }^{- \text {Flox }}$ control mice remained unchanged after the lesion $(n=4)$.

All together these experiments demonstrate that the aberrant crossing ventral to the CC is sufficient to maintain the hopping phenotype and that any dorsal overcrossing due to a gap in EphrinB3 expression has little functional consequence for locomotor coordination.

\section{Discussion}

In this study we have used mouse genetics to functionally demonstrate that the deletion of EphA4 signaling-at the level of the receptor or by blocking its downstream pathways-regionally in the spinal cord is sufficient to generate a hopping locomotor phenotype resembling that of complete EphA4 signaling KOs. In accordance with this finding, we show that the pattern of locomotion is unaffected by the deletion of EphA4 signaling from forebrain neurons despite a pronounced bilateral innervation of the spinal cord. The hopping phenotype is linked to a deletion of EphA4 signaling from excitatory glutamatergic neurons that is accompanied by an increased axonal crossing from excitatory neurons in the ventral spinal cord. The synchronous pattern of activity in complete EphA4 signaling KOs may therefore originate in the rewiring of spinal excitatory Vglut $2^{+}$neurons, placing these neurons as important players in the organization and function of the normal spinal locomotor network.

The hopping phenotype is generated by changes in the local spinal circuitry

One distinctive morphological phenotype in complete EphA4 signaling KOs is the aberrant midline crossing of axons in the spinal cord of both CST neurons and intrinsic spinal interneurons (Dottori et al., 1998; Kullander et al., 2003; Iwasato et al., 2007). The ability to reproduce the hopping phenotype using
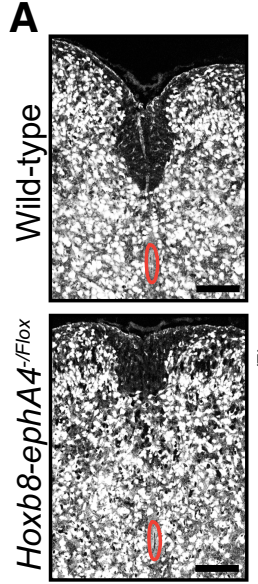

E
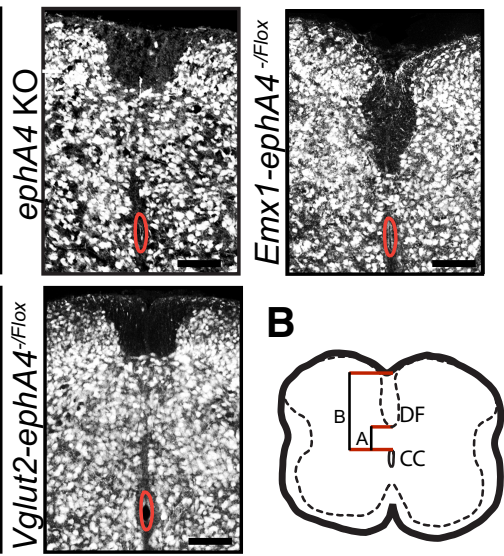

Wild-type
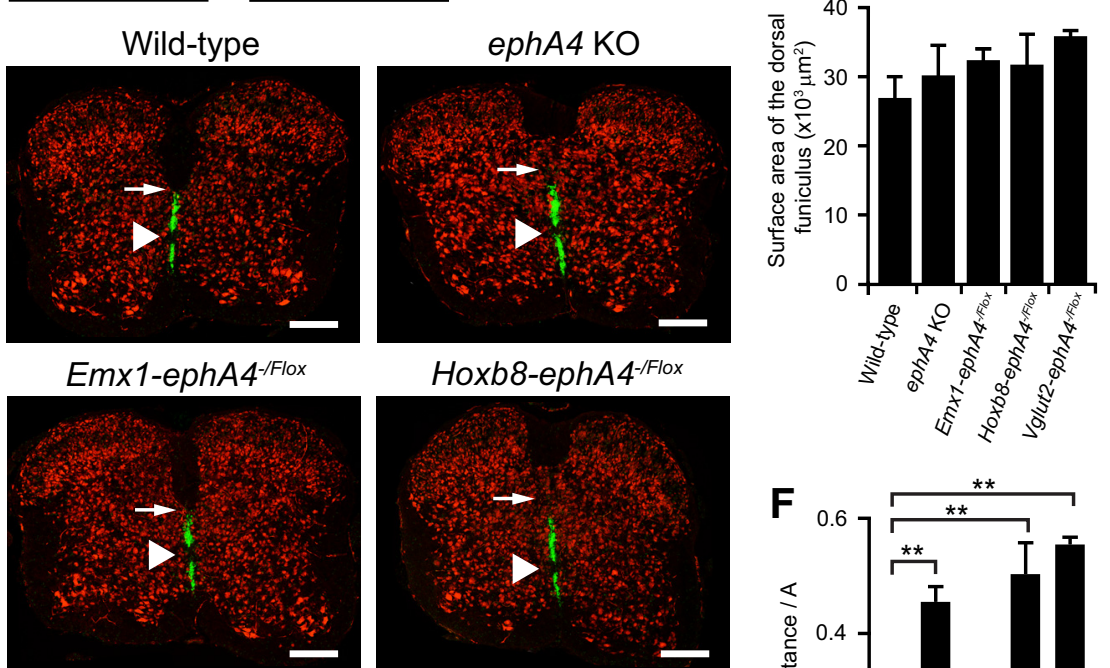

Hoxb8-ephA4//Flox

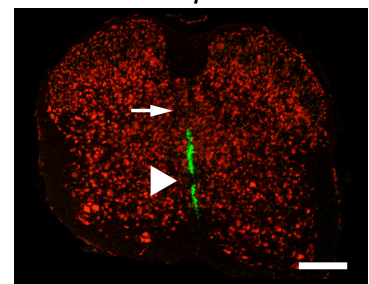

Vglut2-ephA4-/Flox
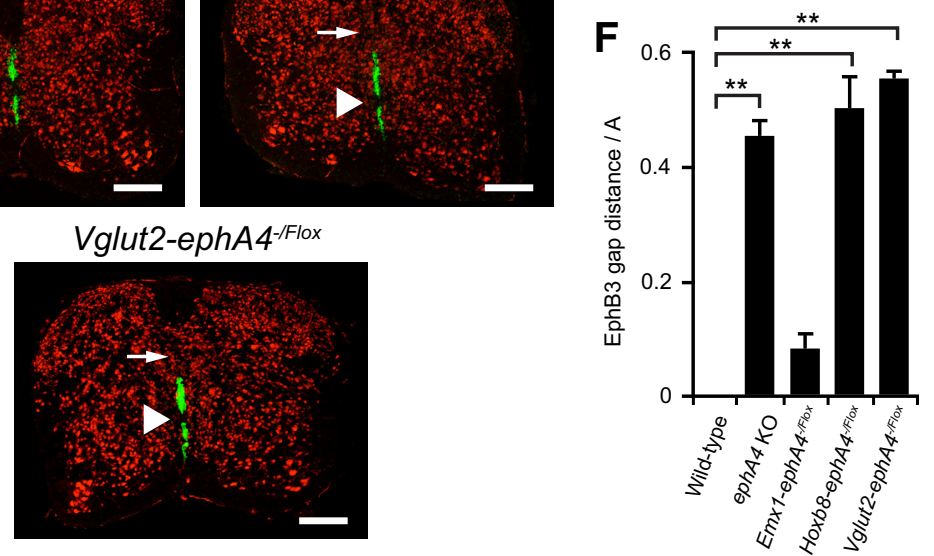

Figure 7. Altered dorsal funiculus (DF) anatomy is correlated with a dorsal gap in EphrinB3 expression. $\boldsymbol{A}$, Confocal images showing the shape of the dorsal commissure in the lumbar spinal cord from newborn (P0-P2) mice. The $C($ is labeled in red. $\boldsymbol{B}$, Schematic figure of the transverse spinal cord showing the calculated distances in $\boldsymbol{C}$ and $\boldsymbol{F}$. A indicates the distance between the $C \boldsymbol{C}$ and the ventral border of the DF, and B indicates the distance between the $C($ and the dorsal edge of the spinal cord. $C$, Bar graph showing the relative distance between the $\mathrm{CC}$ and the ventral edge of the DF normalized to the total distance of the spinal cord dorsal to the CC. Data are represented as mean \pm SD. $\boldsymbol{D}$, Bar graph showing the total area of the DF. Data are represented as mean \pm SD. E, Confocal images showing the EphrinB3 expression in the lumbar spinal cord from P0-P2 animals. White arrowhead indicates the $C($ and the white arrow indicates the gap in the EphrinB3 barrier. $F$, Bar graph showing the dorsal gap in EphrinB3 expression normalized to the total distance from the $C \mathrm{C}$ to the ventral edge of the DF. Data are represented as mean $\pm S D,{ }^{* *} p<0.001$. Scale bars: $A, 100 \mu \mathrm{m} ; \boldsymbol{E}, 200 \mu \mathrm{m}$.

isolated spinal cord preparations from newborn mice pointed to a specific rewiring of spinal networks as responsible for the change in locomotor pattern. However, the CST is not fully developed in newborn mice and it was unclear if the spinal network rewiring is sufficient to produce a hopping gait in adult animals lacking EphA4 signaling or if the bilateral projection of CST axons also contributes to the phenotype. In this study, we demonstrate that the reconfiguration of the spinal locomotor circuitry alone is sufficient to induce the synchronous gait and that the 
Vglut2::Cre; $\alpha-C h n^{-/ F l o x}$
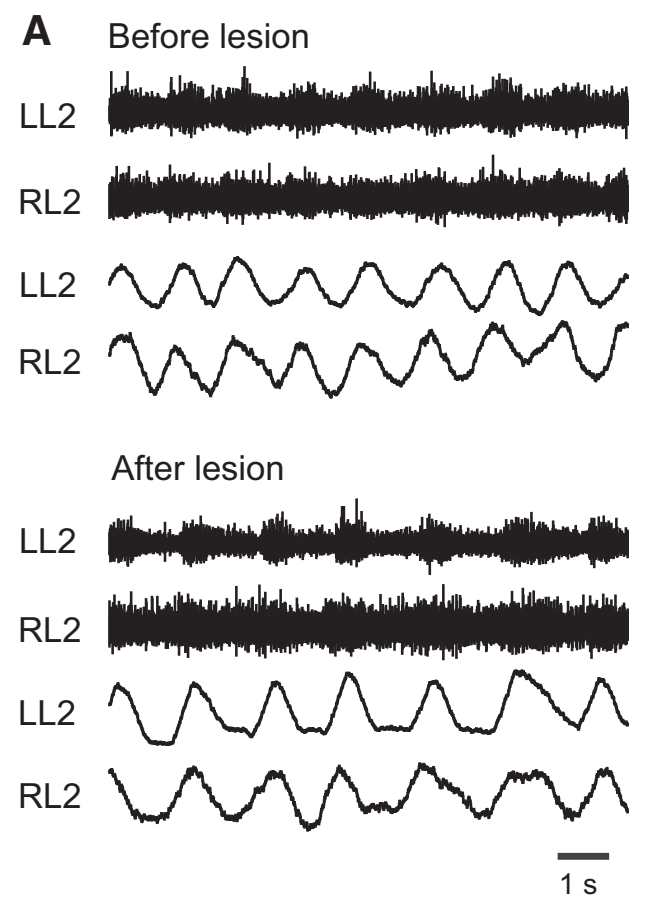
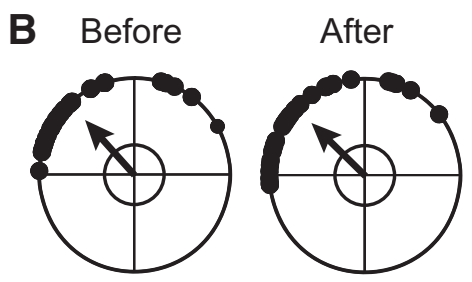

C Before

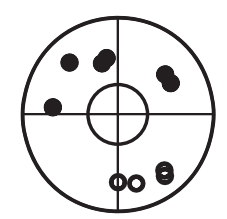

After
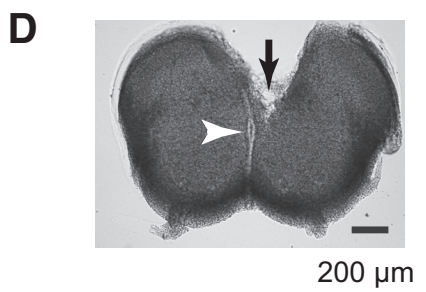

Figure 8. Preservation of left-right VR coupling after lesion of the dorsal commissure in Vglut2::Cre; $\alpha$-Chn ${ }^{-/ \text {Flox }}$ mice. $\boldsymbol{A}$, Locomotor-like activity induced by application of NMDA and 5-HT before (top) and after (bottom) cutting the dorsal commissure in Vglut2::Cre; $\alpha$-Chn ${ }^{-/ F l o x}$ mice. B, Circular plot showing data from the preparation in panel A before (left) and after (right) the dorsal cut. (C) Circular plot with data from all Vglut2::C $\mathrm{Cre} ; \alpha$-Chn ${ }^{-/ F l o x}(N=6)$ and control $\left(\alpha-\mathrm{Chn}^{-/ F l o x}, N=4\right)$ preparations before (left) and after (right) the dorsal cut. Black filled circles correspond to Vglut2:: $\mathrm{Cre} ; \alpha$-Chn ${ }^{-/ F l o x}$ and white circles to controls. D, Anatomical confirmation of the dorsal commissure lesion in the preparation shown in $\boldsymbol{A}$ and $\boldsymbol{B}$. White arrowhead indicates the CC and the black arrow indicates the lesion. Scale bar, $200 \mu \mathrm{m}$.

bilateral innervation of the spinal cord by CST axons does not influence the coordination of overground locomotion. Our results do not exclude an effect of the bifurcating CST axons on skilled locomotion that is known to be under the control of CST neurons (Drew et al., 2002). However, we did not perform more selective tests targeting skilled locomotion or voluntary motor tasks in the present study.

\section{Anatomical changes in the dorsal funiculus have no}

functional consequences for the change in locomotor pattern Another striking morphological phenotype in global EphA4 signaling KOs is the altered shape of the dorsal funiculus (Dottori et al., 1998; Coonan et al., 2001; Kullander et al., 2001; Iwasato et al., 2007). The present study shows that all EphA4 signaling KOs, except the Emx1-EphA4/ $\alpha-C h n \mathrm{KO}$ mice, display a similar change in the shape of the dorsal funiculus, which is accompanied by an increased distance between the CC and the ventral edge of the dorsal funiculus and a gap in the midline expression of EphrinB3 in the most dorsal region of the dorsal commissure. These findings match recent studies showing perturbations of EphA4 signaling in dorsally derived spinal interneuron populations (Paixão et al., 2013). The change in shape of the dorsal funiculus is at least partly caused by an invasion of cells (Restrepo et al., 2011), some of which have specific molecular signatures (Paixão et al., 2013).

The consequence of a dorsal gap in the EphrinB3 barrier is the possibility for both cell-autonomous (removal of EphA4 signaling) and noncell-autonomous (removal of the EphrinB3 barrier) effects when manipulating EphA4 signaling in specific cell populations. Thus, axons from EphA4 competent neurons could poten-

tially cross dorsally due to the EphrinB3 barrier defect. Such added crossing could make it difficult to ascribe the phenotype directly to the neurons lacking EphA4 signaling. However, we have demonstrated, both in this study using the Vglut2::Cre; $\alpha-\mathrm{Chn}^{-/ \text {Flox }}$ mice and in a previous study using the complete EphA4 KO (Restrepo et al., 2011) that the axons responsible for generating the hopping phenotype in EphA4 signaling KOs cross in the ventral commissure and not in the dorsal commissure. These observations suggest that a local noncell-autonomous effect imposed by the gap in EphrinB3 expression dorsal to the CC does not affect our interpretations of the effect on circuit activity linked to the cell-autonomous ablation in specific subsets of neurons in the ventrally located locomotor network.

Identification of glutamatergic neurons responsible for the hopping phenotype Similar to what has been described for the locomotor circuit in the isolated spinal cord preparations from complete EphA4 signaling KOs (Kullander et al., 2003; Iwasato et al., 2007), a left-right alternating locomotor pattern could be observed in vivo in the adult mice, after perturbing the excitatory EphA4 signaling. The alternation was somehow more pronounced in the Vglut2::Cre; $\alpha$-Chn ${ }^{-/ \text {Flox }}$ than in the Vglut2::Cre; ephA4 ${ }^{-/ \text {Flox }}$, possibly because $\alpha$-Chn does not completely capture the full EphA4 population. (Fawcett et al., 2007). Nevertheless, these observations suggest that the commissural interneuron pathways that secure normal left-right alternation (Butt and Kiehn, 2003; Kiehn, 2006; Quinlan and Kiehn, 2007; Talpalar et al., 2013) are conserved in the EphA4 signaling KOs, but are overridden by increased excitation from aberrantly crossing axons derived from the EphA4 ${ }^{+} /$ Vglut ${ }^{+}$population. This suggestion is supported by the observed increase in Vglut2 ${ }^{+}$crossing neurons in the Vglut2specific EphA4 signaling KOs. However, we cannot rule out that this gain of function is accompanied by a loss of function due to a decrease in ipsilateral excitatory drive onto neurons that are crucial for the left-right alternation (Rybak et al., 2013). For example, mice lacking a population of ipsilateral-projecting glutamatergic V2a interneurons, defined by the expression of both Vglut2 and Chx10 (Crone et al., 2008, 2009), show left-right synchronized gait at high locomotor frequencies, presumably due to a decrease in drive onto certain commissural interneurons. Notably, in our study, the EphA4 signaling KOs both in the in vivo experiments in adult animals and in the in vitro experiments in newborn animals showed an alternating phenotype at lower locomotor frequencies. This observation may suggest that if there is a reduced drive of commissural interneurons due to a deletion of EphA4 signaling in Vglut2 ${ }^{+}$neurons, this effect is most pronounced at high locomotor frequencies. Recently we described that inhibitory $\mathrm{V} 0_{\mathrm{D}}$ commissural interneurons are securing leftright alternation at low speeds of locomotion and excitatory $\mathrm{V} 0_{\mathrm{V}}$ commissural interneurons are securing left-right alternation at high speeds of locomotion (Talpalar et al., 2013). Therefore if a 
reduced drive of commissural interneurons is contributing to the hopping phenotype in EphA4 signaling KOs, the activity of the $\mathrm{V}_{\mathrm{V}}$ population should be affected. Since the V2a neurons are supposed to mediate their effect on left-right alternation through the $\mathrm{V}_{\mathrm{V}}$ population (Crone et al., 2008), a selective ablation of EphA4 in V2a neurons should reproduce the hopping gait if this was the sole effect in the Vglut2-specific EphA4 signaling KOs. However, Lhx3::Cre; ephA4 $4^{-/ \text {Flox }}$ mice where EphA4 is eliminated in V2a interneurons do not show any change in the overground locomotor pattern (L. Borgius and O. Kiehn, unpublished observation) suggesting that a decrease in the ipsilateral drive onto commissural interneurons alone is not sufficient to support the phenotype. In contrast, the increased excitatory overcrossing observed after removing EphA4 from Vglut ${ }^{+}$interneurons points to a causal link between the overcrossing and the hopping phenotype.

The simplest explanation for the binding of left-right motor activity into synchrony is that the activity of neurons in the rhythm-generating cores on each side of the spinal cord become synchronized through excitatory aberrant connections between excitatory rhythm-generating neurons, as simulated in modeling studies (Rybak et al., 2013). By inference the increased midline crossing of Vglut $2^{+}$interneurons in the ventral spinal cord suggests that at least some of the EphA $4^{+} / \mathrm{Vglut} 2^{+}$neurons are part of the rhythm-generating core in the locomotor network. Therefore it will be of interest to determine whether the hopping phenotype is generated by known subpopulations of ipsilateral excitatory neurons (e.g., the Shox 2 neurons; Dougherty et al., 2013), or the Di3 (Bui et al., 2013) and Di6 neurons (Dyck et al., 2012) or if the phenotype is mediated by ipsilateral excitatory EphA $4^{+}$populations of yet unknown molecular identity.

\section{References}

Akay T, Acharya HJ, Fouad K, Pearson KG (2006) Behavioral and electromyographic characterization of mice lacking EphA4 receptors. J Neurophysiol 96:642-651. CrossRef

Asante CO, Chu A, Fisher M, Benson L, Beg A, Scheiffele P, Martin J (2010) Cortical control of adaptive locomotion in wild-type mice and mutant mice lacking the ephrin-Eph effector protein alpha2-chimaerin. J Neurophysiol 104:3189-3202. CrossRef Medline

Beg AA, Sommer JE, Martin JH, Scheiffele P (2007) alpha2-Chimaerin is an essential EphA4 effector in the assembly of neuronal locomotor circuits. Neuron 55:768-778. CrossRef Medline

Borgius L, Restrepo CE, Leao RN, Saleh N, Kiehn O (2010) A transgenic mouse line for molecular genetic analysis of excitatory glutamatergic neurons. Mol Cell Neurosci 45:245-257. CrossRef Medline

Bui TV, Akay T, Loubani O, Hnasko TS, Jessell TM, Brownstone RM (2013) Circuits for grasping: spinal dI3 interneurons mediate cutaneous control of motor behavior. Neuron 78:191-204. CrossRef Medline

Butt SJ, Kiehn O (2003) Functional identification of interneurons responsible for left-right coordination of hindlimbs in mammals. Neuron 38:953963. CrossRef Medline

Butt SJ, Lundfald L, Kiehn O (2005) EphA4 defines a class of excitatory locomotor-related interneurons. Proc Natl Acad Sci U S A 102:1409814103. CrossRef Medline

Chédotal A (2011) Further tales of the midline. Curr Opin Neurobiol 21: 68-75. CrossRef Medline

Coonan JR, Greferath U, Messenger J, Hartley L, Murphy M, Boyd AW, Dottori M, Galea MP, Bartlett PF (2001) Development and reorganization of corticospinal projections in EphA4 deficient mice. J Comp Neurol 436:248-262. CrossRef Medline

Crone SA, Quinlan KA, Zagoraiou L, Droho S, Restrepo CE, Lundfald L, Endo T, Setlak J, Jessell TM, Kiehn O, Sharma K (2008) Genetic ablation of V2a ipsilateral interneurons disrupts left-right locomotor coordination in mammalian spinal cord. Neuron 60:70-83. CrossRef Medline

Crone SA, Zhong G, Harris-Warrick R, Sharma K (2009) In mice lacking V2a interneurons, gait depends on speed of locomotion. J Neurosci 29: 7098-7109. CrossRef Medline
Dottori M, Hartley L, Galea M, Paxinos G, Polizzotto M, Kilpatrick T, Bartlett PF, Murphy M, Köntgen F, Boyd AW (1998) EphA4 (Sek1) receptor tyrosine kinase is required for the development of the corticospinal tract. Proc Natl Acad Sci U S A 95:13248-13253. CrossRef Medline

Dougherty KJ, Zagoraiou L, Satoh D, Rozani I, Doobar S, Arber S, Jessell TM, Kiehn O (2013) Locomotor rhythm generation linked to the output of spinal shox2 excitatory interneurons. Neuron 80:920-933. CrossRef Medline

Drew T, Jiang W, Widajewicz W (2002) Contributions of the motor cortex to the control of the hindlimbs during locomotion in the cat. Brain Res Brain Res Rev 40:178-191. CrossRef Medline

Dyck J, Lanuza GM, Gosgnach S (2012) Functional characterization of dI6 interneurons in the neonatal mouse spinal cord. J Neurophysiol 107: 3256-3266. CrossRef Medline

Egea J, Nissen UV, Dufour A, Sahin M, Greer P, Kullander K, Mrsic-Flogel TD, Greenberg ME, Kiehn O, Vanderhaeghen P, Klein R (2005) Regulation of EphA 4 kinase activity is required for a subset of axon guidance decisions suggesting a key role for receptor clustering in Eph function. Neuron 47:515-528. CrossRef Medline

Fawcett JP, Georgiou J, Ruston J, Bladt F, Sherman A, Warner N, Saab BJ, Scott R, Roder JC, Pawson T (2007) Nck adaptor proteins control the organization of neuronal circuits important for walking. Proc Natl Acad Sci U S A 104:20973-20978. CrossRef Medline

Gorski JA, Talley T, Qiu M, Puelles L, Rubenstein JL, Jones KR (2002) Cortical excitatory neurons and glia, but not GABAergic neurons, are produced in the Emx1-expressing lineage. J Neurosci 22:6309-6314. Medline

Herrmann JE, Pence MA, Shapera EA, Shah RR, Geoffroy CG, Zheng B (2010) Generation of an EphA4 conditional allele in mice. Genesis 48: 101-105. CrossRef Medline

Iwasato T, Katoh H, Nishimaru H, Ishikawa Y, Inoue H, Saito YM, Ando R, Iwama M, Takahashi R, Negishi M, Itohara S (2007) Rac-GAP alphachimerin regulates motor-circuit formation as a key mediator of EphrinB3/EphA4 forward signaling. Cell 130:742-753. CrossRef Medline

Iwasato T, Inan M, Kanki H, Erzurumlu RS, Itohara S, Crair MC (2008) Cortical adenylyl cyclase 1 is required for thalamocortical synapse maturation and aspects of layer IV barrel development. J Neurosci 28:59315943. CrossRef Medline

Kiehn O (2006) Locomotor circuits in the mammalian spinal cord. Annu Rev Neurosci 29:279-306. CrossRef Medline

Kjaerulff O, Kiehn O (1996) Distribution of networks generating and coordinating locomotor activity in the neonatal rat spinal cord in vitro: a lesion study. J Neurosci 16:5777-5794. Medline

Kullander K, Mather NK, Diella F, Dottori M, Boyd AW, Klein R (2001) Kinase-dependent and kinase-independent functions of EphA4 receptors in major axon tract formation in vivo. Neuron 29:73-84. CrossRef Medline

Kullander K, Butt SJ, Lebret JM, Lundfald L, Restrepo CE, Rydström A, Klein R, Kiehn O (2003) Role of EphA4 and EphrinB3 in local neuronal circuits that control walking. Science 299:1889-1892. CrossRef Medline

Lundfald L, Restrepo CE, Butt SJ, Peng CY, Droho S, Endo T, Zeilhofer HU, Sharma K, Kiehn O (2007) Phenotype of V2-derived interneurons and their relationship to the axon guidance molecule EphA4 in the developing mouse spinal cord. Eur J Neurosci 26:2989-3002. CrossRef Medline

Paixão S, Balijepalli A, Serradj N, Niu J, Luo W, Martin JH, Klein R (2013) EphrinB3/EphA4-mediated guidance of ascending and descending spinal tracts. Neuron 80:1407-1420. CrossRef Medline

Quinlan KA, Kiehn O (2007) Segmental, synaptic actions of commissural interneurons in the mouse spinal cord. J Neurosci 27:6521-6530. CrossRef Medline

Rabe N, Gezelius H, Vallstedt A, Memic F, Kullander K (2009) Netrin-1dependent spinal interneuron subtypes are required for the formation of left-right alternating locomotor circuitry. J Neurosci 29:15642-15649. CrossRef Medline

Restrepo CE, Lundfald L, Szabó G, Erdélyi F, Zeilhofer HU, Glover JC, Kiehn O (2009) Transmitter-phenotypes of commissural interneurons in the lumbar spinal cord of newborn mice. J Comp Neurol 517:177-192. CrossRef Medline

Restrepo CE, Margaryan G, Borgius L, Lundfald L, Sargsyan D, Kiehn O (2011) Change in the balance of excitatory and inhibitory midline fiber crossing as an explanation for the hopping phenotype in EphA4 knockout mice. Eur J Neurosci 34:1102-1112. CrossRef Medline 
Rybak IA, Shevtsova NA, Kiehn O (2013) Modelling genetic reorganizations in the mouse spinal cord affecting left-right coordination during locomotion. J Physiol 591:5491-5508. CrossRef Medline

Sabatier C, Plump AS, Le Ma, Brose K, Tamada A, Murakami F, Lee EY, Tessier-Lavigne M (2004) The divergent Robo family protein rig-1/ Robo3 is a negative regulator of slit responsiveness required for midline crossing by commissural axons. Cell 117:157-169. CrossRef Medline

Stein E, Zou Y, Poo M, Tessier-Lavigne M (2001) Binding of DCC by netrin-1 to mediate axon guidance independent of adenosine A2B receptor activation. Science 291:1976-1982. CrossRef Medline

Stokke MF, Nissen UV, Glover JC, Kiehn O (2002) Projection patterns of commissural interneurons in the lumbar spinal cord of the neonatal rat. J Comp Neurol 446:349-359. CrossRef Medline

Talpalar AE, Kiehn O (2010) Glutamatergic mechanisms for speed control and network operation in the rodent locomotor CpG. Front Neural Circuits 4: pii:19. CrossRef Medline

Talpalar AE, Bouvier J, Borgius L, Fortin G, Pierani A, Kiehn O (2013) Dual- mode operation of neuronal networks involved in left-right alternation. Nature 500:85-88. CrossRef Medline

Tessier-Lavigne M (2002) Wiring the brain: the logic and molecular mechanisms of axon guidance and regeneration. Harvey Lect 98:103-143. Medline

Wegmeyer H, Egea J, Rabe N, Gezelius H, Filosa A, Enjin A, Varoqueaux F, Deininger K, Schnütgen F, Brose N, Klein R, Kullander K, Betz A (2007) EphA4-dependent axon guidance is mediated by the RacGAP alpha2chimaerin. Neuron 55:756-767. CrossRef Medline

Witschi R, Johansson T, Morscher G, Scheurer L, Deschamps J, Zeilhofer HU (2010) Hoxb8-Cre mice: a tool for brain-sparing conditional gene deletion. Genesis 48:596-602. CrossRef Medline

Yokoyama N, Romero MI, Cowan CA, Galvan P, Helmbacher F, Charnay P, Parada LF, Henkemeyer M (2001) Forward signaling mediated by ephrin-B3 prevents contralateral corticospinal axons from recrossing the spinal cord midline. Neuron 29:85-97. CrossRef Medline

Zar JH (1974) Biostatistical analysis. Edgewood Cliffs, NJ: Prentice-Hall. 